\title{
Sigma-1 Receptor Activation Suppresses Microglia M1 Polarization via Regulating Endoplasmic Reticulum-Mitochondria Contact and Mitochondrial Functions in Stress-induced Hypertension Rats
}

\section{Kokwin Ooi}

Fudan University School of Basic Medical Sciences

Li Hu

Shanghai Jiao Tong University School of Medicine Affiliated Renji Hospital

Yi Feng

Fudan University School of Basic Medical Sciences

Chengzhi Han

Fudan University School of Basic Medical Sciences

\section{Xiaorong Ren}

Fudan University School of Basic Medical Sciences

\section{Xinyi Qian}

Fudan University School of Basic Medical Sciences

\section{Haofeng Huang}

Fudan University School of Basic Medical Sciences

\section{Sijia Chen}

Fudan University School of Basic Medical Sciences

Qi Shi

Fudan University School of Basic Medical Sciences

Hong Lin

Fudan University School of Basic Medical Sciences

Jijiang Wang

Fudan University School of Basic Medical Sciences

\section{Danian Zhu}

Fudan University School of Basic Medical Sciences

\section{Rui Wang}

Tongji University Affilliated Yangpu Hospital: Shanghai Yangpu District Central Hospital Chunmei Xia ( $\nabla$ cmxia@fudan.edu.cn)

Fudan University School of Basic Medical Sciences https://orcid.org/0000-0001-9845-9685 
Research

Keywords: Stress, hypertension, sympathetic nervous system, neuroinflammation, Sigma-1 receptor

Posted Date: January 13th, 2021

DOI: https://doi.org/10.21203/rs.3.rs-142962/v1

License: (c) (i) This work is licensed under a Creative Commons Attribution 4.0 International License. Read Full License

Version of Record: A version of this preprint was published at Molecular Neurobiology on October 2nd, 2021. See the published version at https://doi.org/10.1007/s12035-021-02488-6. 


\section{Abstract}

Background Exposure to stress plays a detrimental role in the pathogenesis of hypertension via noninflammatory pathways. Microglial neuroinflammation in the rostral ventrolateral medulla (RVLM) exacerbates stress-induced hypertension $(\mathrm{SIH})$ by increasing sympathetic hyperactivity. Mitochondria of microglia are the regulators of innate immune response. Sigma-1R ( $\sigma-1 R)$ localizes to the mitochondriaassociated membranes (MAMs) and regulates endoplasmic reticulum (ER) and mitochondria communication, in part through its chaperone activity. The present study aims to investigate the protective role of $\sigma-1 \mathrm{R}$ on microglial-mediated neuroinflammation.

Methods Stress-induced hypertension $(\mathrm{SIH})$ was induced in rats using electric foot shocks and intermittent noise. Arterial blood pressure (ABP), heart rate (HR), and renal sympathetic nerve activity

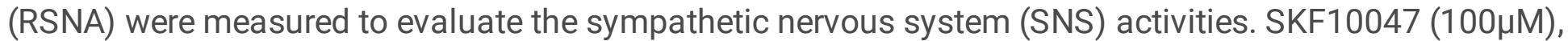
an agonist of $\sigma-1 R$, was administrated to rats, then $\sigma-1 R$ localization and MAMs alterations were detected by immuno-electron microscopy. Mitochondrial calcium homeostasis was examined in primary microglia and/or BV-2 cells. The effect of SKF10047 treatment on the mitochondrial respiratory function of cultured microglia was measured using a Seahorse Extracellular Flux Analyzer. Confocal microscopic images were performed to indicate mitochondrial dynamics.

Results Stress reduced $\sigma-1 \mathrm{R}$ in the MAMs localization, leading to decreased ER-mitochondria contact and IP3R-GRP75-VDAC calcium transport complexes expression in the RVLM of rats. SKF10047 promotes the length and coverage of MAMs in the prorenin treated microglia. Prorenin treatment increases mitoROS levels, and inhibits $\mathrm{Ca}^{2+}$ signaling between the two organelles, therefore negatively affects ATP production in BV2 cells, and these effects are reversed by SKF10047 treatment. We find that mitochondrial hyperfusion and M1 polarization in prorenin-treated microglia. SKF10047 suppresses microglial M1 phenotype and RVLM neuroinflammation, subsequently ameliorates sympathetic hyperactivity in stress-induced hypertensive rats.

Conclusion Sigma-1 receptor activation suppresses microglia M1 polarization and neuroinflammation via regulating endoplasmic reticulum-mitochondria contact and mitochondrial functions in stress-induced hypertension rats.

\section{Background}

Hypertension is a multifactorial disease involving complex interactions among genetic, environmental, vascular, endocrine and neural factors. Chronic exposure to physical or mental stress has been demonstrated to induce and exacerbate stress-induced hypertension $(\mathrm{SIH})[1,2]$. In $\mathrm{SIH}$, multiple mechanisms have been proposed and sympathetic nervous system (SNS) hyperactivity has emerged as a major culprit[3]. Within the cardiovascular sympathetic regulatory centre, local enhanced reninangiotensin system (RAS) activity and augments SNS output, thereafter results in a sustained elevation of arterial blood pressure (BP)[4].This is also confirmed by several animal studies that demonstrate 
microinjection of prorenin (a precursor of RAS cascade) directly activates sympathetic hyperactivity whereas microinjection of prorenin antagonist attenuates the hypertensive effect[5, 6]. Generally, SNS output elevates BP via direct stimulation of sympathetic nerve innervating cardiovascular organs, or via indirect enhancement of release of vasopressinergic hormones involved in salt, volume, and cardiovascular regulation[7].

The central sympathetic drive regulatory centre resides in the central nervous system (CNS), primarily in the rostral ventrolateral medulla (RVLM), the hypothalamic paraventricular nucleus (PVN), and the nucleus tractus solitarius (NTS)[8]. RVLM is the cardiovascular centre that integrates vasomotor input and regulates sympatho-excitatory output. Many factors determine the activity of RVLM neurons, for instance arterial baroreceptors, somatosympathetic afferents, cortex via hypothalamus relay, and carotid chemoreceptors, all of which are associated with the neural mechanism of hypertension[8-10]. More recently, a cause-effect linkage between microglial neuroinflammation and sympatho-excitatory neurons sensitization in the RVLM has been proposed. Several studies highlighted that neuroinflammation at the RVLM foci perturbs the physiological activity of the sympathetic centre, sensitizes the RVLM neurons and thus resulting in sympathetic hyperactivity[10-12]. The pathophysiology of dysregulated sympathetic activity in aggravating hypertension inspires us to study how this process is initiated because it may serve as a rationale for therapeutic intervention.

In the CNS, microglia play pivotal roles in maintaining neural homeostasis and regulating neuroinflammation. Upon sensing stress and/or danger signals, microglia can undergo immunophenotypic transformation to M1/proinflammatory and/or M2/immune-regulatory phenotypes. Activated M1 microglia triggers the assembly of intracellular NLRP3 inflammasome and secretes proinflammatory cytokines (PICs) TNF-a and IL-1 $\beta[13,14]$. Consequently, these PICs can act on target cells, activate adjacent microglia and sensitize neighbouring neurons[15, 16]. Neurotoxic PICs have been demonstrated to sensitize sympathoexcitatory neurons directly or stimulate the release of sensitizing mediators[17]. Our previous study demonstrated the causal relationship between M1-induced neuroinflammation and hypertension by microinjecting minocycline (a selective inhibitor of M1 microglial activation) into the RVLM of SIH rats. The administration of minocycline significantly downregulated microglia-induced neuroinflammation and sympathetic hyperactivity (indicated by reduced plasma norepinephrine) and thus alleviated hypertension[10].

A key link between RAS cascade and M1 microglia activation appears to be cellular oxidative stress, particularly mitochondria-derived reactive oxidative species (mtROS) within RVLM microglia[18]. Mitochondria are regulators of energy metabolism, redox maintenance, and calcium homeostasis therefore modulate many facets of the innate immune response. It is well-established that damaged mitochondria generate cytosolic ROS by mitochondrial electron transport chain and NADPH oxidase (NOX). In our previous studies, we showed that mitochondrial-derived ROS initiates the neuroinflammation process by promoting NLRP3 inflammasome assembly[5]. On the contrary, selective ROS removal has been shown to effectively inhibit M1-derived neuroinflammation and sympathoexcitatory response[10]. Of great importance, the accumulation of ROS-generating 
mitochondria damages mitochondrial DNA and therefore accelerates the neuroinflammation process and favors positive-feedback loop of neuroinflammation as reported in many other neurodegenerative diseases[19-22]. Although the physiological process that initiates this event remains unclear.

Mitochondria-associated membranes (MAMs) is a platform where the endoplasmic reticulum (ER) and mitochondria are physically tethered and MAMs allow dynamic signal transduction between these two organelles[23]. Sigma-1R $(\sigma-1 R)$ is an ER chaperone protein that is primarily resided at the MAMs, numerous evidences report that $\sigma-1 \mathrm{R}$ regulates $\mathrm{Ca}^{2+}$ signalling between ER-mitochondria $[24,25]$. Without $\sigma-1 \mathrm{R}$ activation, the ER-mitochondrial $\mathrm{Ca}^{2+}$ dynamic will be disturbed. Abnormal $\mathrm{Ca}^{2+}$ dynamic has been elucidated to deteriorate mitochondrial bioenergetic and trigger mitochondrial ROS production[26]. Additionally, the depletion of $\sigma-1 \mathrm{R}$ alters mitochondrial homeostasis and leads to the pathogenesis of numerous neurodegenerative diseases [27-29]. Consistent with these observations, our present study aims to reveal the neuroprotective of $\sigma-1 R$ activation, which might suppress microglia $M 1$ polarization via regulating MAMs-dependent $\mathrm{Ca}^{2+}$ dynamic and mitochondrial functions, and thus alleviating neuroinflammation in stress-induced hypertension. Our present study implies that the manipulation of $\sigma-1 \mathrm{R}$ could be a promising target for the prevention or treatment of hypertension.

\section{Methods}

\section{Chemicals and antibodies}

The following chemicals were used: recombinant human prorenin (ab93266) was from Abcam (Cambridge, MA) and SKF10047 (1079) was purchased from Tocris Bioscience (Bristol, UK). Artificial cerebrospinal fluid (aCSF) (CZ0530) was purchased from Leagene (Leagene Biotechnology, Beijing, China). ATP Assay Kit (S0026), Mito-Tracker Green (C1048), ER-Tracker Red (C1041) were purchased from Beyotime (Shanghai, China). The following antibodies were used: rabbit anti-sigma-1R (\#15168-1AP) were from Proteintech; sheep monoclonal antiserum against prorenin/renin (GTX79677, Gene Tex, San Antonio, TX); rabbit anti-CD86 (\#91882), anti-CD206 (\#91992), anti-MFN2 (\#9482), anti-OPA1 (\#67589), anti-Drp1 (\#8570), anti-GAPDH (\#5174), anti-GRP75 (\#3539), anti-ASC (\#13833), anti-Caspase1 (D7F10) and IL-1 $\beta$ (D3U3E) were from Cell Signalling Technology (Beverly, MA); mouse anti-IP3R (\#SC377518) was purchased from Santa Cruz Biotechnology (Santa Cruz, CA); rabbit monoclonal antiserum against TTC11/Fis1 (ab156865), mouse monoclonal to $\beta$-actin (ab8226), VDAC1 (\#ab14734 1:5000 for WB); rabbit polyclonal antiserum against NLRP3 (ab214185) were from Abcam. Goat antimouse IgG H\&L (Alexa Fluor 488) (ab150113); Goat anti-Rabbit IgG H\&L (Alexa Fluor 594) (ab150080); Donkey Anti-Rabbit IgG H\&L (Alexa Fluor 647) (ab150075); Donkey Anti-Rabbit IgG H\&L (HRP) (ab6802) and Donkey Anti-Mouse IgG H\&L (HRP) (ab6820) were purchased from Abcam (Cambridge, UK).

\section{Experimental design}


Experimental 1: To explore the expression and the regulatory roles of $\sigma-1 \mathrm{R}$ in SIH in vivo, Adult SpragueDawley rats (male, 8 weeks old, 250-300 g) were randomly assigned to three groups (6 rats each): (i) normotensive (Ctrl); (ii) stress-induced hypertensive (SIH); (iii) SIH + SKF10047 (a prototype $\sigma-1 \mathrm{R}$ agonist). The rats were housed under 12 -h light/dark cycle condition in a temperature-controlled room at $24^{\circ} \mathrm{C}$ with standard food and tap water ad libitum. SKF10047 was used to activate $\sigma-1 \mathrm{R}$ in SIH rats, specifically, $100 \mu \mathrm{M}$ intracisternal infusion into $\mathrm{SIH}$ rats was carried out by osmotic minipump once daily for 1 week (from stress day 8 to day 15).

Experimental 2: To identify the roles of $\sigma-1 \mathrm{R}$ in mitochondrial associated endoplasmic reticulum membranes (MAMs) as well as to investigate the antagonistic effects of $\sigma-1 \mathrm{R}$ activation in proinflammatory phenotype (M1) switching. The primary microglial cells were obtained and cultured ex vivo. Primary microglial cells were seeded and grown for 7 days in DMEM supplemented with $10 \%$ (vol/vol) serum (basal conditions). Next, they were divided into 3 groups: (i) control (Ctrl, vehicle); (ii) prorenin (20

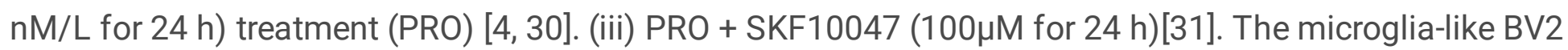
cells in each cohort were used in the case that the primary microglia were inadequate in the abovementioned experiments.

\section{Animal preparation}

Eight weeks old male Sprague-Dawley (SD) rats (260-300g) and 1-old-day SD rats were used for this study and purchased from the Animal Laboratory Center of Fudan University. All experiments were performed on 110 adult male SD rats and 501 -old-day SD rats, with a mortality rate of $5 \%$ due to intolerance of brain surgery, or death during stress. We have described the groups and the design of the $\mathrm{SIH}$ model for this study. In short, rats were subjected to electric foot shock with noises. They were put in a Plexiglas chamber $(26 \mathrm{~cm} \times 21 \mathrm{~cm} \times 26 \mathrm{~cm})$ with a grid floor made of stainless-steel rods $(0.3 \mathrm{~cm}$ diameter, spaced $1.0 \mathrm{~cm}$ apart), connected to a shock generator. A series of foot shocks, comprised of $0.5-\mathrm{mA}$ intensity of 1-s duration with shock interval of $4 \mathrm{~min}$, were controlled and generated electronically. Synchronously, noises (range 88-98 dB) produced by a buzzer were given as a conditioned stimulus. After acclimatization for a few times, the stressed group rats were subjected to stress for $2 \mathrm{~h}$ twice daily for 15 consecutive days. The control group underwent sham stress. During the days of stress, BP and HR (heart rate) in conscious rats were measured by non-invasive tail-cuff system (ALC-NIBP, Shanghai Alcott Biotech) every 4 days after treatments according to our previous study[32]. To adapt to the BP recording procedure, the animals were placed on the warm platform for $30 \mathrm{~min}$. BP was measured in a proper environment (RT, lightning, and noise-free atmosphere). BP measurements were replicated for at least 3 times, and the average value was obtained.

\section{Cell culture}

As described previously [33], primary cultures of microglial cells were prepared from the medulla oblongata covering the RVLM, which was removed from 1-day-old Sprague-Dawley rats after 
decapitation. The RVLM was identified according to the atlas of Watson and Paxinos[34]. Both sides of the RVLM (about 1.5- to 2.5-mm lateral to the midline and medial to the spinal trigeminal tract) were collected using micropunches with a 1-mm inner diameter burr. Next, Hanks balanced salt solution dissecting medium containing glucose, bovine serum albumin (BSA), and HEPES, as well as $0.025 \%$ trypsin was used to incubate the minced tissue at $37^{\circ} \mathrm{C}$ for $20 \mathrm{~min}$. Then, cells were cultured at a density of $3 \times 10^{5}$ cells $/ \mathrm{cm}^{2}$ in Dulbecco's modified Eagle's medium (DMEM)/F12 medium (Biochrom, Berlin, Germany) supplemented with 10\% fetal bovine serum (FCS; Biochrom), $0.1 \mathrm{mg} / \mathrm{mL}$ streptomycin and 100 $\mathrm{U} / \mathrm{mL}$ penicillin in poly-L-lysine-coated $75 \mathrm{~cm}^{2}$ culture flasks. After 3 days, the culture medium was removed and replaced with fresh medium and kept at $37^{\circ} \mathrm{C}$ in a humidified $95 \% \mathrm{O}_{2} / 5 \% \mathrm{CO}_{2}$ incubator. On day 9 , the cells were re-suspended after centrifugation $(150 \times \mathrm{g}$ for $10 \mathrm{~min})$ [35]. The purity of cultured microglia was $>90 \%$ when evaluated by flow cytometry.

\section{BP measurements and RSNA recording}

We recorded RSNA when the general procedures for acute experiments were ready, the RSNA recording method was adapted from our previous study[5]. In anesthetized rats (urethane $800 \mathrm{mg} / \mathrm{kg}$ and achloralose $40 \mathrm{mg} / \mathrm{kg}$ ip), the right formal artery was catheterized for the recording of BP and HR using a Powerlab system (AD Instruments). Next, the left renal sympathetic nerves were exposed, identified and carefully dissected free from the surrounding connective tissue, and then they were placed on a pair of silver recording electrodes (Teflon 786500, A-M Systems Inc., Sequim, WA). Both the nerves and the electrodes were covered with Kwik-Sil gel (World Precision Instruments). The nerve signal was amplified (bandwidth: $100-3,000 \mathrm{~Hz}$ ) with a Grass P55C preamplifier (model P 18D, Grass Instruments). The amplified discharge was monitored on a storage oscilloscope (model $121 \mathrm{~N}$; Tektronix, Beaverton, OR), and the maximum value of RSNA was calculated after the rat was overdosed by narcotic euthanasia (200 $\mathrm{mg} / \mathrm{kg}$ ). Baseline RSNA was taken as a percentage of maximum after the background noise was subtracted. The background noise level was recorded 15-20 min after the rat was euthanized using the unit conversion of the PowerLab Chart system.

\section{Implantation of Intracisternal Osmotic Minipump}

The procedures were performed as described in our previous study[5]. Briefly, the rats were anesthetized as above-mentioned. After a midline dorsal neck incision, the dura was perforated with a 22-gauge steel needle, and following cerebrospinal fluid leakage, a PE-5 catheter (Clay Adams, Sparks, MD) was advanced $5 \mathrm{~mm}$ into the cisterna magna and sealed to the dura with tissue glue. The outer end of the catheter was connected to a micro-osmotic minipump (Alzet 1007D, Durect Co., Cupertino, CA). The SKF10047 $(100 \mu \mathrm{M})$ microinjection was delivered by osmotic minipump. Rats were used in normal physiological condition after the operation in subsequent experiments. After SKF10047 microinjections, rats were treated with 1000 units of penicillin through muscle injection to prevent infection. 


\section{Transmission electron microscopy}

Transmission electron microscopy analysis was performed as stated in previous publications $[10,32,36]$. The cells were collected in the EP tubes after the cell medium was decanted, the cells were fixed with $2 \%$ paraformaldehyde plus $2.5 \%$ glutaraldehyde in $0.1 \mathrm{M}$ phosphate buffer, $\mathrm{pH} 7.2-7.4$, and then they were osmicated, rinsed with phosphate buffer, dehydrated, and embedded in epoxy resin, which then they were allowed to polymerize for $24 \mathrm{~h}$ at $70{ }^{\circ} \mathrm{C}$. Blocks containing microglia were sectioned using an ultramicrotome (Ultracut; Leica) at 70-80 nm. Thin sections were collected on grids and stained with uranyl acetate and lead citrate. Grids were examined under a transmission electron microscope $(\mathrm{H}-700$; Hitachi, Tokyo, Japan) at $80 \mathrm{kV}$.

\section{Flow Cytometry analysis of M1/M2 phenotype}

For M1 phenotype classification via flow cytometry analysis, primary microglial cells were cultured at a density of $3 \times 10^{5}$ cells/ $\mathrm{cm}^{2}$ and starved with DMEM/DF12 with $2 \%$ FBS overnight. As described

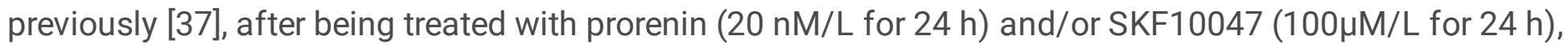
microglial cells were harvested followed by centrifugation at $300 \times \mathrm{g}$ for $5 \mathrm{~min}$ at $4{ }^{\circ} \mathrm{C}$. Cells were surfacestained for $45 \mathrm{~min}$ in the darkroom with fixation and permeabilization buffer as per the manufacturer's instructions, then they were incubated with PE-conjugated anti-mouse CD86 (0.125 $\mu \mathrm{g} / \mathrm{test}$; eBioscience, Waltham, MA, USA) and APC-conjugated anti-rat CD206 (10 $\mu \mathrm{l} / 106$ cells; R\&D, MN, USA). Finally, the microglial cells were washed twice with PBS and were resuspended in $500 \mu \mathrm{PBS}$. Data were analyzed using Flowing software v2.5.1 (FACS Calibur running CellQuest Pro; Becton Dickinson, UK) and the percentage of positive cells was documented.

\section{Immunofluorescent Staining and Confocal Microscopic Imaging}

As stated in previous literature[32], immunofluorescent staining was detected by laser confocal microscopy. To determine the co-localization of pro-inflammatory phenotype (M1) microglia CD86 with $\sigma-1 R, M F N 2, O P A 1$, and NLRP3, the rats were euthanized with excessive pentobarbital sodium (200 $\mathrm{mg} / \mathrm{kg}$, ip), then $0.9 \% \mathrm{NaCl}$ solution and $4 \%$ paraformaldehyde in $0.1 \mathrm{M}$ phosphate buffer solution (PBS) were used to perfuse the aorta through a constant flow pump. RVLM sections were collected and post-fixed with $4 \%$ paraformaldehyde for $4 \mathrm{~h}$. The RVLM sections were then dehydrated in $20 \%-30 \%$ sucrose until they sank to the bottom of the solution, respectively. Free-floating $30 \mu \mathrm{m}$ coronal sections were cut on a cryostat (Microm, Germany) and were frozen at $-20^{\circ} \mathrm{C}$ floated in $0.01 \mathrm{M} \mathrm{PBS}$ (pH 7.4). These coronal sections containing the RVLM were mounted on slides after 3-5 min washes and then incubated with $0.3 \%$ Triton X-100 for 30 min followed incubation by $5 \%$ horse serum for $1 \mathrm{~h}$ at $37^{\circ} \mathrm{C}$ to block nonspecific protein. RVLM coronal sections were incubated with primary antibody overnight at $4{ }^{\circ} \mathrm{C}$. The Goat anti-mouse IgG H\&L (Alexa Fluor 488) (ab150113); Goat anti-Rabbit IgG H\&L (Alexa Fluor 594) 
(ab150080); Donkey Anti-Rabbit IgG H\&L (Alexa Fluor 647) (ab150075) secondary antiserum were used as secondary antibody. $\sigma-1 \mathrm{R}, \mathrm{MFN2}$, OPA1, prorenin, and NLRP3 colocalization with M1 phenotype microglial marker CD86 was investigated in vivo. For in vitro experiment, Primary microglial cells were cultured on glass cover slips and treated with prorenin and /or SKF10047 for 24h. After being washed with PBS, cells were fixed with 4\% PFA for 10 min, then incubated with blocking buffer (5\% BSA and $0.1 \%$ Triton X-100; Gentihold, Beijing, China) for $30 \mathrm{~min}$, primary antibodies $(\sigma-1 \mathrm{R}, 1: 400)$ overnight at $4{ }^{\circ} \mathrm{C}$. Subsequently incubated with correspond fluorochrome-conjugated secondary antibodies at room temperature for $1 \mathrm{~h}$. immunoreactivity manifested as specific green or red fluorescence. Images were captured using confocal microscope (LSM800, Carl Zeiss Microscopy Ltd, Cambridge, MA).

\section{Western blot analysis}

Microglia isolated from the RVLM tissue of each mouse were homogenized in lysis buffer with $1 \%$ NP40, $1 \mathrm{mM}$ PMSF. In brief, equivalent amounts of protein (20 $\mu \mathrm{g}$ per lane) were loaded and separated by SDS/PAGE in 8\%-15\% gradient gels and were transferred to PVDF membrane (Invitrogen, Carlsbad, CA, USA). The membranes were blocked with $5 \%$ nonfat milk solution in tris-buffered saline with $0.1 \%$ Triton $\mathrm{X}-100$ (TBST, Invitrogen, Carlsbad, CA, USA) for $1 \mathrm{~h}$, and then incubated overnight at $4{ }^{\circ} \mathrm{C}$ with the primary $\sigma-1 \mathrm{R}$ antibody (1:1000), CD86 antibody (1:1000), MFN2 antibody (1:1000), Fis1 antibody (1:1000), Drp1 antibody (1:1000), OPA1 antibody (1:1000), IP3R antibody (1:1000), GRP75 antibody (1:1000), VDAC antibody (1:1000), NLRP3 antibody (1:1000), ASC antibody (1:1000), Caspase-1 antibody (1:1000), IL-1ß antibody (1:1000), GAPDH antibody (1:1000) and $\beta$-actin (1:1000) antibody dilutions in TBST. After that, the membranes were washed and incubated with secondary antibody for $1 \mathrm{~h}$ at room temperature. The amount of detected protein was assessed using ECL detection reagents (WBKLS0050; Millipore), and the immunoactivity band was detected by a fully automatic chemiluminescence image analysis system (Tanon-5200; Tanon Science \& Technology, Shanghai, China). The levels of target proteins were normalized to GAPDH or $\beta$-actin, which served as a loading control. Each immunostaining band was visualized and quantitated by an analysis software named Image $J$ (National Institutes of Health, Boston, MA, USA).

\section{Oxygen Consumption Rate (OCR)}

Oxygen consumption rates were measured by an XFe96 extracellular flux analyzer (Seahorse Biosciences, North Billerica, MA). In short, cells were seeded in a 96-well plate in DMEM/DF12 medium at a density of $3 \times 105$ cells/ $\mathrm{cm} 2$ before measurement. One hour before measurements, the cell culture medium was replaced with $180 \mu \mathrm{l} \mathrm{FX}$ assay medium (\#102365-100, Seahorse Biosciences) composed of $10 \mathrm{mM}$ glucose, $1 \mathrm{mM}$ pyruvate, and $2 \mathrm{mM}$ glutamine at $\mathrm{pH} 7.4$, and then incubated at $37^{\circ} \mathrm{C}$ under normal atmosphere. XF cell Mito Stress Test Kit (\#103015-100, Seahorse Biosciences) was used in the FX assay medium. Measurements were obtained at $37^{\circ} \mathrm{C}$. This assay procedure included mixing ( $\left.3 \mathrm{~min}\right)$, waiting period ( $2 \mathrm{~min}$ ) and measurement ( $3 \mathrm{~min})$. Oxygen consumption rate (OCR) was analyzed using wave 
software provided by Seahorse Biosciences. Basal respiration, maximal respiration, and proton leak were determined by XF Cell Mito Stress Test Generator that was provided by Seahorse Bioscience.

\section{Real-Time RT-PCR}

Total RNA of RVLM was extracted using extraction reagent and converted into first-strand cDNA (TaKaRa Biotechnology, Co., Ltd., Dalian, China) in accordance with manufacturer's instructions. The mRNAs of CD86, CD206, $\sigma-1 R$, IL-1 $\beta$, TNF-a, IL-10, TGF- $\beta$, NLRP3, ASC, Caspase-1, $\beta$-actin and GAPDH were analyzed by quantitative real-time PCR. The sequences of primers were listed in Table $\mathrm{S1}$. For normalization and relative quantification, the housekeeping gene $\beta$-actin/GAPDH was used as the internal standard reference gene. The relative quantification of gene expression was expressed as fold-change via normalization against $\beta$-actin by using the $2^{\Delta \Delta}$ CT method. Three tissues of three rats were used in each group. A 1-2 fold change versus normal controls was considered significant.

Table S1

The primer sequences used of the target genes measured

\begin{tabular}{|c|c|c|}
\hline \multirow[t]{2}{*}{ Gene } & \multicolumn{2}{|l|}{ Primer } \\
\hline & Forward & Reverse \\
\hline IL-1 $\beta$ & 5'-TGACCGAGATGTGGAGG-3' & 5'-GAATGGCAATGATGAGGT-3' \\
\hline TNF-a & 5'-TTCTCATTCCTGCTCGTGG-3' & 5'-CCGCTTGGTGGTTTGC-3' \\
\hline TGF- $\beta$ & 5'-ATGCCGCCCTCGGGGCTGCGGCTGC-3' & 5'-TACGGCGGGAGCCCCGACGCCGACG-3' \\
\hline IL-10 & 5'-CTGCTATGTTGCCTGCTCTT-3' & 5'-GGTCAGTCGGTCTGGGTGTA-3' \\
\hline CD86 & 5'-CACCCGAAACCTAAGATGT-3' & 5'-TGAAAGAGAGAGGCTGTTGGA-3' \\
\hline$\sigma-1 R$ & 5'-TGAGAGACCACGGGGACCTG-3' & 5'-GCACCACACTGACTCTTCCATTC-3' \\
\hline NLRP3 & 5'-TTTCTGACCCTCGTAGTCTATCCA-3' & 5'-GGCTGTGGACAATGGGAAGG-3' \\
\hline ASC & 5'CCCCATAGACCTCACTGATAAA-3' & 5'-GCCCATAGCCTTCCCGCACT-3' \\
\hline Caspase1 & 5'-CTTCTTTGTTCTGCGTCTGC-3' & 5'-ATTTGTCCCTTAGAAACAGC-3' \\
\hline$\beta$-actin & 5'-ACAGCTTCTTTGCAGCTCCTTCG-3' & 5'-ATCGTCATCCATGGCGAACTGGTG-3' \\
\hline GAPDH & 5'-GTGCTGAGTATGTCGTGGAGTCTAC-3' & 5'-GGCGGAGATGATGACCCTTTTGG-3' \\
\hline
\end{tabular}

\section{Mitochondrial ROS measurement}

Mitochondrial ROS were measured following the manufacturer's instructions of MitoSOX Red kit (Thermo Fisher Scientific). Briefly, treated cells cultured on glass were washed and incubated with $0.5 \mu \mathrm{M}$ MitoSOX. After reaction, cells were washed and observed by an inverted fluorescence microscope for 


\section{Endoplasmic Reticulum (ER) $\left[\mathrm{Ca}^{2+}\right]_{E R}$ and Mitochondrial $\left[\mathrm{Ca}^{2+}\right]_{\mathrm{M}}$ Measurement}

Cultured primary microglial cells were transfected with the mitochondria matrix targeted $\mathrm{Ca}^{2+}$ fluorescent sensor CEPIA4 and ER lumen-targeted $\mathrm{Ca}^{2+}$ fluorescent sensor GECO protein[36, 38, 39]. For mitochondrial $\mathrm{Ca}^{2+}$ measurements, primary microglial cells were transiently transfected with the plasmids pCMVR-CEPIA4mt targeted to the mitochondrial matrix $48 \mathrm{~h}$ before the experiments. To measure $\mathrm{Ca}^{2+}$ of ER, primary microglial cells were loaded with ER lumen-targeted $\mathrm{Ca}^{2+}$ fluorescent sensor GECO protein in normal culture media and/or prorenin-treated culture media for 2 or 3 days before imaging. For the capture of subcellular ER and mitochondrial $\mathrm{Ca}^{2+}$ signals, time-lapse or snapshot images of $\mathrm{Ca}^{2+}$ signals were obtained using confocal microscope (LSM800, Carl Zeiss Microscopy Ltd, Cambridge, MA) equipped with a Lambda DG4 illumination system (Sutter Instrument). Cells were illuminated at $440 \mathrm{~nm}$ (440AF21; Omega Optical) through a 455DRLP dichroic mirror, and emission was collected alternatively at $480 \mathrm{~nm}$ (480BP10; Omega Optical) and $535 \mathrm{~nm}$ (535AF26; Omega Optical), using a cooled, 12-bit CCD camera (CoolSnap HQ; Ropper Scientific). Cells were washed briefly at room temperature with intracellular-like medium (ICM) containing: $125 \mathrm{mM} \mathrm{KCl}, 19 \mathrm{mM} \mathrm{NaCl}, 10 \mathrm{mM} \mathrm{Hepes} \mathrm{(pH}$ 7.3 with $\mathrm{KOH}$ ), and $1 \mathrm{mM}$ EGTA, $10 \mathrm{mM}$ glucose, $\mathrm{pH}$-adjusted at 7.45 with $\mathrm{NaOH}$.

\section{Statistical analysis}

Data were expressed as means \pm SEM (standard error of mean). Statistical differences between the SIH rats treated with SKF10047, prorenin and/or SKF10047 treated microglia were analyzed by Student's $t$ test. For comparison between multiple groups, one-way or two-way analysis of variance with repeated measurements was used to determine the differences between groups. When an ANOVA was significant, post hoc testing of differences between groups was performed using the least significant difference (LSD) test. $P<0.05$ indicated that the differences were statistically significant. Statistical analysis was performed using GraphPad Prism (GraphPad Software, Version 6.0, La Jolla, CA, United States).

\section{Results}

\section{Stress significantly reduces $\sigma-1 R$ expression and upregulates M1 microglial activation and neuroinflammation in the RVLM of rats.}


Recent studies have shown that sigma-1 receptor agonists can inhibit neuroinflammation[40, 41]. $\sigma-1 \mathrm{R}$ is highly expressed in neurons as well as in microglia. The above statement inspires us to investigate the profiles of $\sigma-1 \mathrm{R}$ and microglial M1 activation during stress-induced hypertension (SIH). Activated microglia are mainly divided into the pro-inflammatory $\mathrm{M} 1$ type $\left(\mathrm{CD} 86^{+}\right)$and anti-inflammatory $\mathrm{M} 2$ type $\left(C D 206^{+}\right)$. Activated M1 microglia secretes pro-inflammatory cytokines TNF- $\alpha$ and IL-1 $\beta$, whereas the M2 type promotes repair and regeneration by releasing anti-inflammatory TGF- $\beta$ and IL-10[42]. We analysed the expressions of $\sigma-1 \mathrm{R}$ and CD86 (M1 microglia marker) in the RVLM of rats using double immunofluorescence, immunoblotting, and/or transmission electron microscopy, respectively. The double immunofluorescence staining showed that the $\sigma-1 R$ was extensively expressed in a variety of cells in rat RVLM. $\sigma-1 R$ and CD86 colocalization was decreased in the stressed rats than that of the control group (Fig. 1A-B, $P<0.01, n=6$ ). The immunoblot revealed that in the RVLM region of stressed rats, the expression of $\sigma-1 R$ protein was 2.8 times lower, while the protein expression of CD86 stressed rats was 3.5 times higher than that of control rats (Fig. $1 C-D, P<0.01, n=6$ ). This quantitative analysis revealed the negative correlational relationship between the $\sigma-1 \mathrm{R}$ expression and $\mathrm{M} 1$ polarization under stress.

We need to confirm the effectiveness of SKF10047 (specific $\sigma-1 R$ agonist) in M1 activation. qRT-PCR of RVLM data showed that compared to the control group, the stressed rats showed notable elevation of expression of TNF- $a$ and IL-1 $\beta$ (Fig. 1E-F, $P<0.01, n=6$ ). On the other hand, microinjection of SKF10047 into the RVLM area decreased the expression of TNF- $a$ and IL-1 $\beta$ (Fig. $1 E-F, P<0.01, n=6$ ). The results of western blotting showed that stress upregulated the expression of $\mathrm{CD} 86^{+}$and downregulated the expression of $\mathrm{CD}^{206^{+}}$, whereas SKF10047 treatment reversed these results (Fig. $1 \mathrm{G}-\mathrm{H}, \mathrm{P}<0.05, \mathrm{n}=6$ ). Furthermore, immunofluorescent staining indicated that SKF10047 treatment attenuated stress-induced neuroinflammation as showed by the diminished ratio of $\mathrm{CD} 86^{+} / \mathrm{CD} 206^{+}$when comparing to the stressed group (Fig. 1I-J, $P<0.05, n=6$ ). The results indicate that $\sigma-1 R$ activation effectively inhibits the polarization of M1 phenotype microglia and thereby reduces neuroinflammation in the RVLM area of stressed rats.

\section{Stress decreases the expression of $\sigma-1 R$ at the mitochondrial-associated endoplasmic reticulum membrane (MAMs), stimulating dissociation of MAMs.}

Using immune transmission electron microscopy observation, we found that $\sigma-1 \mathrm{R}$ was predominantly distributed and expressed at the mitochondrial-associated endoplasmic reticulum membrane (MAMs) (Yellow arrows in Fig. 2A). The stressed group exhibited lesser amount of $\sigma-1 \mathrm{R}$ tethered at the MAMs compared to the control group. Conversely, the SKF10047 treatment increased its expression. In the second experiment, we used Mito-Tracker and ER-Tracker to label mitochondria and endoplasmic reticulum and combined with the $\sigma-1 \mathrm{R}$ staining method to detect the MAMs/ $\sigma-1 \mathrm{R}$ co-localization. Compared to the fluorescence density of the control group, prorenin significantly suppressed Mito-ER contact (MAMs association) and MAMs/ $\sigma-1 R$ co-localization (Fig. 2B-D, $P<0.05, n=6$ ). On the contrary, 
SKF10047 treatment increased the expression of $\sigma-1 R$, improved Mito-ER contact, and MAMs/ $\sigma-1 R$ colocalization (Fig. 2B-D, $P<0.05, n=6$ ). This result reveal that $\sigma-1 R$ was expressed at the mitochondrialassociated endoplasmic reticulum membrane (MAMs) and its localization was decreased in stress rats.

\section{Transmission electron microscopy showed that stress stimulates dissociation of MAMs. Activation of $\sigma-1 R$ promotes the formation and association of MAMs.}

More direct evidence is required to clarify the role of stress in suppressing $\sigma-1 \mathrm{R}$ and causing MAMs dissociation. In this context, we quantified the MAMs association by using transmission electron microscopy, whereby the MAMs contact length and MAMs coverage (ratio of MAMs contact length to mean mitochondrial circumference) were measured. We found that stress (artificially elicited by prorenin) significantly reduced MAMs length and MAMs coverage compared to the control group (Fig. 3A-C, $\mathrm{P}<$ $0.05, \mathrm{n}=6$ ). However, upon SKF10047 stimulation, MAMs contact length and MAMs coverage were significantly increased (Fig. $3 A-C, P<0.05, n=6$ ). This result indicates that stress stimulates MAMs dissociation, whereas $\sigma-1 R$ activation promotes and stabilizes the structural formation of Mito-ER contact, hence enhancing MAMs association.

\section{4. $\sigma$-1R activation stabilizes MAMs and IP3R-GRP75-VDAC transport complexes, subsequently rescues the MAMs- dependent $\mathrm{Ca}^{2+}$ signalling between the two organelles.}

$\sigma-1 \mathrm{R}$ is a chaperone protein that stabilizes MAMs' structure and it binds to the $\mathrm{Ca}^{2} \rrbracket$ channel Ins $(1,4,5)$ $\mathrm{P}_{3} \mathrm{Rs}$ (IP3R) at the MAMs, thereby preventing the ubiquitination and degradation of IP3R[43, 44]. GRP75 and VDAC microdomains are then recruited to bind to the IP3R, forming IP3R-GRP75-VDAC transport complexes which mechanistically connect the outer membranes of ER and mitochondria to facilitate $\mathrm{Ca}^{2+}$ uptake by mitochondria[24, 45]. When the MAMs were dissociated, we hypothesized that this could directly alter $\mathrm{Ca}^{2+}$ signalling between the ER and the mitochondria. Western blotting revealed that in the RVLM of stressed rats, the protein expressions of IP3R-GRP75-VDAC were significantly reduced compared to the control group, whereas $\sigma-1 R$ agonist treatment significantly upregulated the IP3R-GRP75-VDAC expression (Fig. 4A-B, $\mathrm{P}<0.01, \mathrm{n}=6$ ). Furthermore, we also studied the MAMs-mediated $\mathrm{Ca}^{2+}$ dynamics by comparing the $\mathrm{Ca}^{2+}$ concentration in the 2 organelles using mitochondria-targeted genetically encoded $\mathrm{Ca}^{2+}$ indicators (CEPIA4) and endoplasmic reticulum-targeted genetically encoded $\mathrm{Ca}^{2+}$ indicators (GECO). We observed that endoplasmic reticulum $\mathrm{Ca}^{2+}$ concentration had increased and the intramitochondrial $\mathrm{Ca}^{2+}$ concentration had decreased (Fig. 4C-D) after prorenin stimulation for 24 hours. Conversely, with SKF10047 treatment for 6 hours, the endoplasmic reticulum $\mathrm{Ca}^{2+}$ concentration was found to decrease, and most importantly mitochondrial $\mathrm{Ca}^{2+}$ concentration was increased in prorenin- 
cultured primary microglia (Fig. 4C-D). These results strengthen the hypothesis that $\sigma-1 \mathrm{R}$ activation stabilizes MAMs and IP3-GRP75-VDAC transport complexes, subsequently rescue the MAMs-dependent $\mathrm{Ca}^{2+}$ signalling between the two organelles in stressed settings.

\section{Stress disturbed mitochondrial respiration homeostasis and increased concentration of mtROS. $\sigma$-1R activation resumes ATP production and mtROS in microglia.}

Manipulation or failure of mitochondrial bioenergetics has been shown to induce neuroinflammation and neurodegenerative diseases like Parkinson's and Alzheimer's disease[46, 47]. We aimed to determine if stress would exert any effect on mitochondrial respiration and bioenergetics. We cultured BV2 microglia cell lines, treated them with prorenin and/or SKF10047, the effects of SKF10047 on mitochondrial aerobic respiration of microglia were detected by Seahorse cell metabolometer. The results showed that under FCCP and oligomycin-induced mitochondrial stress states, OCR was significantly increased in microglia. Prorenin induced a reduced OCR, suggesting that prorenin suppresses mitochondrial respiration. By contrast, SKF10047 $(100 \mu \mathrm{M})$ induced a dramatically increased OCR. Prorenin reduced MG ATP synthesis and decreased maximal respiration and respiratory potential. While the reduced ATP concentration was rescued and significantly elevated after SKF10047 treatment (Fig. 5A-E, $P<0.05, n=6$ ). There are numerous evidences indicate that dysfunctional mitochondria releasing ROS (mtROS), of which the mitoROS can act as upstream activators of NLRP3 inflammasome. We found that the concentration of mtROS was significantly upregulated in the prorenin group, and its concentration was attenuated by SKF10047 treatment (Fig. 5F-G, $P<0.05, n=6$ ). These results indirectly strengthen our hypothesis that stress-inhibited MAMs-dependent $\mathrm{Ca}^{2+}$ dynamics disturbed mitochondrial bioenergetics, which causes accumulation of dysfunctional mitochondria that release mtROS. On the contrary, SKF10047 treatment significantly attenuated mtROS production.

\section{Stress stimulates mitochondrial hyperfusion, Resumption of $\mathrm{Ca}^{2+}$ dynamics by $\sigma-1 \mathrm{R}$ activation protects against mitochondrial hyperfusion and thus prevents microglial M1- polarization.}

Mitochondria are dynamic organelles that can manipulate their morphology in response to cellular stress and physiological signal via fusion and fission machinery $[48,49]$. Mitochondrial membrane dynamics influence and often orchestrate various cellular functions, for instance cellular $\mathrm{Ca}^{2+}$ transfer[50], apoptosis[51] and immune response[52]. We were interested to explore if stress-inhibited MAMsdependent $\mathrm{Ca}^{2+}$ signalling would result in an imbalance of mitochondrial dynamics, of which might contribute to microglia M1 polarization. Transmission electron microscopy visualized that prorenin 
induced mitochondrial hyperfusion, as represented by the fused and elongated mitochondria in comparison to the control group (Fig. 6A, left). The super-resolution microscopy also disclosed that mitochondria were transformed into enlarged and network form due to hyperfusion (Fig. 6A middle and right). Interestingly, SKF10047 treatment reduced prorenin-induced hyperfusion as represented by the relatively normal mitochondrial size and shape. Fission is primarily governed by fission protein (Drp1 and Fis1), whereas fusion relies on the fusion protein (MFN2 and OPA1)[23]. Western blot results showed that the expressions of MFN2 and OPA1 in the RVLM area of stressed rats were remarkably upregulated, but the expressions of Drp1 and Fis1 were decreased (Fig. 6B-C, P< 0.05, $n=6$ ). In contrast, SKF10047 treatment effectively suppressed the expressions of MFN2 and OPA1 and increased the expressions of Drp1 and Fis1 (Fig. 6B-C, $\mathrm{P}<0.05, \mathrm{n}=6$ ). The colocalization analysis indicated that MFN2 (Fig. 6D-F, $\mathrm{P}<$ $0.05, \mathrm{n}=6$ ) and OPA1 (Fig. 6G-I, $\mathrm{P}<0.05, \mathrm{n}=6$ ) expression in the activated microglia (CD86) of the stressed group were increased in comparison to that of the control group. Most importantly, SKF10047 administration decreased the colocalization of CD86 with MFN2 and OPA1 respectively (Fig. 6D-I, P< $0.05, n=6$ ). These results reinforce that stress stimulates mitochondrial hyperfusion via upregulation of OPA1 and MFN2 protein expression. Imbalanced fusion-fission machinery causes the accumulation of dysfunctional mitochondria and therefore activates microglia M1 polarization.

\section{SKF10047 treatment reduces the ratio of M1/M2 polarization and NLRP3 inflammasomes activation in prorenin-treated primary microglia.}

In our previous research, we proved that stress activates the assembly of NLRP3 inflammasome, leading to the maturation of proinflammatory cytokine (IL-1 $\beta$ ) and thus microglial M1 polarization[5]. We had already demonstrated that stress-inhibited MAMs-dependent $\mathrm{Ca}^{2+}$ signalling led to decreased ATP production and mitochondrial hyperfusion, both of which contributed to the accumulation of dysfunctional mitochondria. Mitochondrial dysfunction, leading to the release of mitochondrial ROS (mtROS) and DNA (mt-DNA) into the cytosol, are critical upstream events in initiating NLRP3 inflammasome activation[53, 54].

To analyse the quantitative change of M1 microglia in response to stressed condition and $\sigma-1 \mathrm{R}$ treatment. We have isolated primary microglia $(\mathrm{CD} 11 \mathrm{~b}+)$ and confirmed that the purity of primary microglia was greater than $90 \%$ using flow cytometry analysis (Fig. 7A). These primary microglia were subjected to prorenin and SKF10047 treatment respectively. Fluorescence-activated cell sorting (FACS) analysis was then used to quantified M1 (CD86+) and M2 (CD206+) microglia. FACS revealed that prorenin induced immunophenotypic transformation of $M 2$ to $M 1$ microglia, whereas $\sigma-1 R$ activation preferentially reduced immunophenotypic transformation of M2 to M1 microglia (Fig. 7B-C, $P<0.05, n=5$ ).

We also analyzed $\sigma-1 R, C D 86$, CD206, pro-inflammatory cytokines (TNF-a, IL- $\beta$ ) and anti-inflammatory cytokines (IL-10, TGF- $\beta$ ) by Real-Time PCR. In the prorenin group, the mRNA expression of $\sigma-1 \mathrm{R}$ was decreased, and the mRNA expression of CD86 and pro-inflammatory cytokines (TNF-a, IL- $\beta$ ) were 
increased (Fig. 7D, $P<0.05, n=5$ ). $\sigma-1 R$ activation decreased mRNA expression of CD86, TNF- $a$, and IL- $\beta$ (Fig. 7D, $P<0.05, n=5$ ).

Additionally, immunofluorescence staining indicated that NLRP3 protein expression was elevated by 3fold as compared to the control group, and $\sigma-1 R$ activation downregulated the NLRP3 protein expression (Fig. 7F, $P<0.05, n=5$ ). We also demonstrated that the colocalization of CD86/NLRP3 was elevated in the prorenin group, whereas the colocalization was downregulated after SKF10047 treatment (Fig. 7E-G, $P<0.05, n=5)$. Collectively, $\sigma-1 R$ activation prevents NLRP3 activation and M1 microglia polarization.

\section{In-vivo studies showed that $\sigma$-1R activation preventing NLRP3 activation and M1 microglia polarization.}

In this in-vivo experiment, parallel with the results of in-vitro studies, RT-PCR and western blot assays showed that stress downregulated mRNA and protein expressions of $\sigma-1 \mathrm{R}$, and stress upregulated mRNA and protein expressions of NLRP3, ASC, and Caspase-1 (Fig. 8A-C, $P<0.05, n=6$ ). Contrarily, SKF10047 treatment increased the expressions of $\sigma-1 R$ and decreased the expressions of NLRP3, ASC and Caspase1 (Fig. 8A-C, $P<0.05, n=6$ ). M1 microglia (CD86+), NLRP3 positive cells, and their colocalization were measured by double immunofluorescent staining (Fig. 8D). NLRP3 expressions were approximately elevated by 4 -fold by stress stimulation, and they were downregulated after $\sigma-1 R$ activation (Fig. $8 E, P<$ $0.05, n=6$ ). Most importantly, the colocalization of CD86/NLRP3 was elevated in the stressed group, whereas the colocalization was downregulated after SKF10047 treatment (Fig. 8F, P<0.05, $n=5$ ).

\section{9. $\sigma-1 R$ activation suppresses sympathetic hyperactivity and hypertension in rats.}

Here, we aimed to unravel the protective effect of $\sigma-1 R$ activation in RVLM on alleviating sympathetic hyperactivity in stressed rats. In this context, sympathetic activities were quantified in the form of mean arterial pressure (MAP), renal sympathetic nerve activity (RSNA), and \%Max RSNA from baseline RSNA(\%MAX). The results showed that the MAP, RSNA and \%MAX were considerably elevated in the stressed group in comparison to those in the control group (Fig. 9A-C, $P<0.05, n=6$ ). The application of $\sigma-1 R$ agonist SKF10047 reduced MAP to the proximity of basal levels in the control group, and \% MAX RSNA was significantly downregulated by approximately $15 \%$ Max from the peak level of $40 \%$ Max in stress-treated rats (Fig. 9A-C, $P<0.05, n=6$ ). Therefore, we concluded that $\sigma-1 R$ activation suppresses sympathetic hyperactivity in vivo.

\section{Discussion}

In our preliminary studies, we demonstrated that ROS-generating dysfunctional mitochondria play crucial roles in triggering microglia M1 polarization and enhancing sympathetic hyperactivity at the RVLM. The 
main purpose of this research was to investigate the plausible role of $\sigma-1 R$ in protecting the RVLM from neuroinflammation via promoting MAMs association and structural stability of IP3Rs-GRP75-VDAC complexes. Accordingly, the neuroprotective role of $\sigma-1 R$ was evaluated by microinjection of SKF10047 (selective $\sigma-1 \mathrm{R}$ agonist) into the RVLM of stressed rats. However, there is no direct evidence to support that these effects are attributed to microglia since multiple cell types exist in the RVLM. To address this issue, we culture primary microglia and treated them with prorenin (to simulate stress condition) and SKF10047. Our major findings were: (1) Stress suppressed $\sigma-1 R$ activation, thereafter triggered MAMs dissociation and inhibited MAMs-dependent $\mathrm{Ca}^{2+}$ dynamics between ER and mitochondria. (2) The prorenin-inhibited MAMs-dependent $\mathrm{Ca}^{2+}$ dynamics induced mitochondrial ROS overproduction, reduced mitochondrial ATP production, stimulated mitochondrial hyperfusion, thereby resulting in the accumulation of dysfunctional mitochondrial. (3) Stress induces NLRP3 inflammasome assembly and PICs release, stimulated immunophenotypic polarization of M1 microglia. (4) $\sigma-1 R$ activation by SKF10047 treatment resumed the stress-inhibited $\mathrm{Ca}^{2+}$ dynamics at the MAMs by promoting MAMs association and structural stability of IP3Rs-GRP75-VDAC complexes. (5) $\sigma-1 \mathrm{R}$ activation significantly suppressed RVLM neuroinflammation and ameliorated sympathetic hyperactivity in stressed rats. (Schematic Fig. 10)

Possible regulatory role of $\sigma-1 \mathrm{R}$-mediated MAMs-dependent $\mathrm{Ca}^{2+}$ dynamic on inflammation was proposed several years ago when Wang et al. found that $\sigma-1 R-K O$ retinal Müller cells manifest elevated ROS, whereas activation of $\sigma-1 \mathrm{R}$ reduces ROS formation and inflammation[26]. In this study, we demonstrated for the first time that chronic physical stress is enough to suppress $\sigma-1 R$ activation, which consequently induces downstream M1 polarization. In addition, we demonstrated that stress-induced sympathetic hyperactivity can be alleviated by activation of $\sigma-1 R$, this information provides foundation for further studies regarding the neuroprotective role of $\sigma-1 R$ activation.

Recent studies indicate that $\sigma-1 \mathrm{R}$ regulates mitochondrial membrane dynamics, ATP production and redox states via modulating MAMs-dependent $\mathrm{Ca}^{2+}$ transfer. Failure of $\sigma-1 \mathrm{R}$ to orchestrate these functions likely leads to mitochondrial dysfunction, robust ROS generation, and thus initiates inflammation[23]. Based on our results, the effect of $\sigma-1 \mathrm{R}$ on MAMs-dependent $\mathrm{Ca}^{2+}$ dynamic can be explained from 2 aspects. Firstly, using TEM and immunofluorescence assay, we demonstrated that $\sigma-1 R$ improves MAMs association as indicated by upregulated MAMs contact length and coverage. Besides, the upregulated colocalization of $\sigma-1 R$ (purple fluorescence) at Mito-ER colocalization indicate possible role of $\sigma-1 R$ in mediating biophysical interaction between Mito-ER. We therefore speculated the aggregation of $\sigma-1 \mathrm{R}$ at the MAMs contact site can help to facilitate the MAMs-dependent $\mathrm{Ca}^{2+}$ dynamics according to cellular needs, possibly by acting as an anchor allowing mitochondria and ER to stay connected and preventing them from drifting away. Secondly, it has been demonstrated that $\sigma-1 \mathrm{R}$ activation is essential for stabilizing the ultrastructure of IP3Rs, a specific $\mathrm{Ca}^{2+}$ transport protein at the MAMs[24]. Cytosolic freely floating mature IP3Rs have been shown to undergo rapid ubiquitination and degradation $[43,44]$. The presence of activated $\sigma-1 R$ provides a platform for binding of free-floating IP3Rs, consequently, the stabilized IP3Rs can recruit GRP75 and VDAC proteins to form IP3R-GRP75-VDAC 
transport complexes ensuring proper $\mathrm{Ca}^{2+}$ fluxes from the ER to the mitochondria[24, 45]. Our data show satisfactory agreement with this previous hypothesis as $\sigma-1 R$ activation by SKF10047 was successful in increasing in the protein expression IP3-GRP75-VDAC transport components. Moreover, the increased intramitochondrial $\mathrm{Ca}^{2+}$ concentration coupled with decreased intra-ER $\mathrm{Ca}^{2+}$ concentration by $\sigma-1 \mathrm{R}$ activation strengthen the role of $\sigma-1 \mathrm{R}$ in facilitating MAMs-dependent $\mathrm{Ca}^{2+}$ flux kinetics. Taken together, we have demonstrated that stress suppresses $\sigma-1 R$ activation, thereby stimulating MAMs dissociation and inhibiting MAMs-dependent $\mathrm{Ca}^{2+}$ dynamics.

A key unanswered question remains about how does the inhibited $\mathrm{Ca}^{2+}$ dynamic lead to mitochondrial dysfunction. $\sigma-1 \mathrm{R}$-mediated $\mathrm{Ca}^{2+}$ transport into mitochondria might play roles in determining ATP production and metabolic state of mitochondria. Specifically, the accumulated $\mathrm{Ca}^{2+}$ directly activates TCA cycle by binding to $\mathrm{Ca}^{2+}$ dependent pyruvate dehydrogenase phosphatase, a-ketoglutarate dehydrogenases and isocitrate dehydrogenases $[55,56]$. Stimulation of these $\mathrm{Ca}^{2+}$-sensitive enzymes increases the rate of TCA cycles and thus NADH availability and ATP production[45]. Here, we demonstrated that stress (prorenin in vitro) inhibited $\mathrm{Ca}^{2+}$ dynamics at the MAMs, thus impairing mitochondrial ATP production and causing overproduction of mitoROS. Treatment of the $\sigma-1 R$ agonist SKF10047 counteracted the imbalanced $\mathrm{Ca}^{2+}$ homeostasis caused by stress, suggesting that refilling of the intracellular $\mathrm{Ca}^{2+}$ serves a protective role against mitochondrial oxidative phosphorylation deterioration.

Another aspect regarding the perturbation of mitochondrial homeostasis is disturbed mitochondrial membrane dynamics. In physiologic condition, mitochondria perform basal fusion to allow the exchange of matrix content of a damaged mitochondrion with healthy mitochondria, whereas fission allows segregation of damaged content (e.g., mutated DNA/ROS) to daughter organelle, aiming for degradation of damaged content via autophagy[57]. In line with this notion, our data showed that mitochondrial hyperfusion as visualized by TEM and increased expression DRP1 and MFN2 proteins. The rationale behind the fusion phenomenon is that fusion allows the $\mathrm{Ca}^{2+}$-deprived mitochondria to redistribute and share $\mathrm{Ca}^{2+}$, thus maximizing the efficiency of mitochondrial ATP production[58,59]. The hyperfusion process may seem to be beneficial at glance, however, this compensatory mechanism actually results in elongated and swollen mitochondria which render autophagy ineffective in engulfing this large hyperfused network and therefore spare them from autophagic degradation[60]. As time passes, defective mitophagy causes the accumulation of damaged mitochondria and ROS generation, consequently promoting a pro-inflammatory microenvironment which can trigger NLRP3 inflammasome activation[53, 61]. From another perspective, Nakahira and colleague demonstrated that inhibition of mitophagy stimulates excessive ROS which later damages proximity mitochondrial DNA (mtDNA), then causes the release of damaged mtDNA into cytosol and eventually triggers NLRP3 activation[62, 63]. Parallel with these findings, our results confirmed that elevated mitochondrial ROS production in stressed group was attenuated by $\sigma-1 \mathrm{R}$ activation (supplementary figure). However, it should be noted that we did not demonstrate the sole causal relationship between mitochondrial hyperfusion and mitochondrial ROS 
production because ROS can be generated by ER, lysosome, peroxisome and other organelle, future investigation is certainly warranted in this regard.

NLRP3 inflammasome is a multi-protein complex. After NLRP3 inflammasome is assembled and activated, the Caspase- 1 and ASC within the activated NLRP3 inflammasome in turn promotes the maturation of proinflammatory cytokines (PICs) which are capable to polarize primary microglia to activated M1 microglia [54, 64]. Our study has shed light on $\sigma-1 \mathrm{R}$-mediated $\mathrm{Ca}^{2+}$ dynamics as crucial upstream regulators for NLRP3 activation and microglial M1 polarization. Interestingly, a recent studies study conducted by Zhou et.al reveal MAMs provide locations for NLRP3 inflammasome assembly, it is therefore plausible that other MAM-resident proteins (e.g: TPM, PERK, MFN1) might involve in the interaction of inflammation response[53, 65].

Our results underlined that $\sigma-1 \mathrm{R}$ activation attenuated the augmented sympathetic activity in stressed rats. RSNA was used as a parameter because RVLM directly governs RNSA output and increased RSNA contributes to the rise of blood pressure via urinary sodium water retention and activation of RAAS cascade[66]. Clinically, catheter-based renal sympathetic denervation has been shown to substantially reduce $\mathrm{BP}$ in resistant hypertension, but data shows there is progressive re-generation of sympathetic activity that limits the effectiveness of this technique[67]. Our data shows that $\sigma-1 R$ activation can as centrally-acting sympatholytic via attenuating neuroinflammation at the RVLM (see Fig. 10, content figure). Collectively, the validation of the crosstalk between stress, $\sigma-1 \mathrm{R}$ and M1-polarization extends our understanding of the unique pathogenesis of stress-induced hypertension and open an avenue for therapeutic opportunities via modulation of $\sigma-1 R$.

\section{Conclusions}

Collectively, our results implied that $\sigma-1 \mathrm{R}$ activation suppresses microglia M1 polarization and neuroinflammation via regulating endoplasmic reticulum-mitochondria contact and mitochondrial functions in stress-induced hypertension rats.

\section{Limitations}

There are several technical limitations of this study deserve comment. Firstly, under normal conditions, $\sigma-1 \mathrm{R}$ are widespread on the neurons, astrocytes, microglia and oligodendrocytes as well. Therefore, further effects need to be observed to specifically interfere the $\sigma-1 R$ in microglia. Secondly, the interaction among the above-mentioned cell types in the regulation of sympathetic activity in hypertension settings needs to be clarified.

\section{List Of Abbreviations}




\begin{tabular}{|c|c|}
\hline $\mathrm{SIH}$ & stress-induced hypertension \\
\hline RVLM & rostral ventrolateral medulla \\
\hline CNS & central nervous system \\
\hline SNS & sympathetic nervous system \\
\hline RSNA & renal sympathetic nerve activity \\
\hline MAMs & mitochondria-associated endoplasmic reticulum membranes \\
\hline ER & endoplasmic reticulum \\
\hline PICs & pro-inflammatory factors \\
\hline$\sigma 1-\mathrm{R}$ & sigma-1 receptor \\
\hline Drp1 & dynamin-related factors \\
\hline Fis1 & fission protein 1 \\
\hline MFN2 & mitofusion 2 \\
\hline OPA1 & optic atrophy 1 \\
\hline LAMP2 & lysosomal-associated membrane protein 2 \\
\hline aCSF & artificial cerebrospinal fluid \\
\hline PBS & phosphate buffer saline \\
\hline BSA & bovine serum albumin \\
\hline Ang $\otimes$ & angiotensin $\otimes$ \\
\hline RAS & renin-angiotensin system \\
\hline ROS & reactive oxygen species \\
\hline HR & heart rate \\
\hline HRV & heart rate variability \\
\hline ABP & arterial blood pressure \\
\hline MAP & mean arterial pressure \\
\hline $\mathrm{NADPH}$ & nicotinamide adenine dinucleotide phosphate \\
\hline NE & norepinephrine \\
\hline NLRP3 & NLR family pyrin domain containing 3 \\
\hline ASC & apoptosis-associated specklike protein containing a caspase recruitment domain \\
\hline IP3R & inositol 1,4,5-triphosphate receptor \\
\hline
\end{tabular}


GRP75 glucose-regulated protein 75

VDAC voltage-dependent anion channel

TNF-a tumer necrosis factor-a

TEM transmission electron microscope

OCR oxygen consumption rate

\section{Declarations}

\section{Ethics approval and consent to participate}

All experimental protocols were approved by the Fudan University Animal Care Committee, and all operations in this study were conformed to the guidelines of the Institutional Ethics Committee.

\section{Consent for publication}

All the listed authors have approved the delivery of the manuscript for publication.

\section{Availability of data and materials}

All relevant data and materials are within the manuscript.

\section{Competing interests}

The authors declare that they have no competing interests.

\section{Funding}

This work was supported by the Chinese National Natural Science

Foundation 81770423 to CMX and No.31271215 to DNZ. The funders had no role in study design, data collection and analysis, decision to publish, or preparation of the manuscript.

\section{Authors' contributions}

LH conducted the experiments of BP measurement and microinjection in vivo. XYQ, HFH, XRR, HL, SJC, $\mathrm{CZH}$ and QS participated in the immunoblot, immunohistochemistry staining and PCR experiments. $\mathrm{LH}$ and Kokwin Ooi acquired and analyzed the data. CMX and RW conceived the study and participated in experiment design and coordination. CMX, YF, JJW and DNZ contributed reagents/materials/analysis 
tools. Kokwin Ooi, CMX and LH drafted, polished and revised the manuscript. All authors have been read and approved the final manuscript.

\section{Acknowledgement}

We thank Dr. Weizhong Wang and his research team (Department of Physiology and Center of Polar Medical Research, Navy Military Medical University, Shanghai, China.) for their excellent technical support in RSNA recording experiment. We thank for Dr. Yu Liang and Dr. Chao Zhang's technical support in double-stained immunofluorescent images taken by ZEISS Lattice SIM (ZEISS microscopy customer center, Shanghai).

\section{References}

1. Zimmerman RS, Frohlich ED: Stress and hypertension. J Hypertens Supp/ 1990, 8:S103-107.

2. Liu MY, Li N, Li WA, Khan H: Association between psychosocial stress and hypertension: a systematic review and meta-analysis. Neurol Res 2017, 39:573-580.

3. Young CN, Davisson RL: Angiotensin-II, the Brain, and Hypertension: An Update. Hypertension (Dallas, Tex : 1979) 2015, 66:920-926.

4. Shi P, Grobe JL, Desland FA, Zhou G, Shen XZ, Shan Z, Liu M, Raizada MK, Sumners C: Direct proinflammatory effects of prorenin on microglia. PLoS One 2014, 9:e92937.

5. Hu L, Zhang S, Ooi K, Wu X, Wu J, Cai J, Sun Y, Wang J, Zhu D, Chen F, Xia C: Microglia-Derived NLRP3 Activation Mediates the Pressor Effect of Prorenin in the Rostral Ventrolateral Medulla of Stress-Induced Hypertensive Rats. Neurosci Bull 2020, 36:475-492.

6. Dong T, Chen J-W, Tian L-L, Wang L-H, Jiang R-D, Zhang Z, Xu J-B, Zhao X-D, Zhu W, Wang G-Q, et al: Role of the renin-angiotensin system, renal sympathetic nerve system, and oxidative stress in chronic foot shock-induced hypertension in rats. In International journal of biological sciences, vol. 11. pp. 652-663; 2015:652-663.

7. Guyenet PG: The sympathetic control of blood pressure. Nat Rev Neurosci 2006, 7:335-346.

8. Barrett KE, Barman SM, Yuan JXJ, Brooks H: Ganong's Review of Medical Physiology, 26e. 2019.

9. Biancardi VC, Son SJ, Ahmadi S, Filosa JA, Stern JE: Circulating angiotensin II gains access to the hypothalamus and brain stem during hypertension via breakdown of the blood-brain barrier. Hypertension 2014, 63:572-579.

10. Du D, Hu L, Wu J, Wu Q, Cheng W, Guo Y, Guan R, Wang Y, Chen X, Yan X, et al: Neuroinflammation contributes to autophagy flux blockage in the neurons of rostral ventrolateral medulla in stressinduced hypertension rats. Journal of neuroinflammation 2017, 14:169-169.

11. Wu KLH, Chan SHH, Chan JYH: Neuroinflammation and oxidative stress in rostral ventrolateral medulla contribute to neurogenic hypertension induced by systemic inflammation. Journal of Neuroinflammation 2012, 9:212. 
12. Gao L, Wang W, Li Y-L, Schultz HD, Liu D, Cornish KG, Zucker IH: Sympathoexcitation by central ANG II: Roles for AT1 receptor upregulation and NAD(P)H oxidase in RVLM. American Journal of Physiology-Heart and Circulatory Physiology 2005, 288:H2271-H2279.

13. Orihuela R, McPherson CA, Harry GJ: Microglial M1/M2 polarization and metabolic states. British Journal of Pharmacology 2016, 173:649-665.

14. Tang Y, Le W: Differential Roles of M1 and M2 Microglia in Neurodegenerative Diseases. Mol Neurobio/ 2016, 53:1181-1194.

15. Schäfers M, Sorkin L: Effect of cytokines on neuronal excitability. Neuroscience Letters 2008, 437:188-193.

16. Yao K, Zu H-b: Microglial polarization: novel therapeutic mechanism against Alzheimer's disease. Inflammopharmacology 2020, 28:95-110.

17. Xue B, Thunhorst Robert L, Yu Y, Guo F, Beltz Terry G, Felder Robert B, Johnson Alan K: Central Renin-Angiotensin System Activation and Inflammation Induced by High-Fat Diet Sensitize Angiotensin II-Elicited Hypertension. Hypertension 2016, 67:163-170.

18. Choi S-H, Aid S, Kim H-W, Jackson SH, Bosetti F: Inhibition of NADPH oxidase promotes alternative and anti-inflammatory microglial activation during neuroinflammation. Journal of neurochemistry 2012, 120:292-301.

19. Hamacher-Brady A, Brady NR: Mitophagy programs: mechanisms and physiological implications of mitochondrial targeting by autophagy. Cellular and Molecular Life Sciences 2016, 73:775-795.

20. Kerr JS, Adriaanse BA, Greig NH, Mattson MP, Cader MZ, Bohr VA, Fang EF: Mitophagy and Alzheimer's Disease: Cellular and Molecular Mechanisms. Trends in neurosciences 2017, 40:151-166.

21. Hu Z-L, Sun T, Lu M, Ding J-H, Du R-H, Hu G: Kir6.1/K-ATP channel on astrocytes protects against dopaminergic neurodegeneration in the MPTP mouse model of Parkinson's disease via promoting mitophagy. Brain, Behavior, and Immunity 2019, 81:509-522.

22. Fang EF, Hou Y, Palikaras K, Adriaanse BA, Kerr JS, Yang B, Lautrup S, Hasan-Olive MM, Caponio D, Dan $X$, et al: Mitophagy inhibits amyloid- $\beta$ and tau pathology and reverses cognitive deficits in models of Alzheimer's disease. Nature Neuroscience 2019, 22:401-412.

23. Giacomello M, Pyakurel A, Glytsou C, Scorrano L: The cell biology of mitochondrial membrane dynamics. Nat Rev Mol Cell Biol 2020, 21:204-224.

24. Hayashi T, Su TP: Sigma-1 receptor chaperones at the ER-mitochondrion interface regulate $\mathrm{Ca}(2+)$ signaling and cell survival. Cell 2007, 131:596-610.

25. Mori T, Hayashi T, Hayashi E, Su TP: Sigma-1 receptor chaperone at the ER-mitochondrion interface mediates the mitochondrion-ER-nucleus signaling for cellular survival. PLoS One 2013, 8:e76941.

26. Wang J, Shanmugam A, Markand S, Zorrilla E, Ganapathy V, Smith SB: Sigma 1 receptor regulates the oxidative stress response in primary retinal Müller glial cells via NRF2 signaling and system xc(-), the $\mathrm{Na}(+)$-independent glutamate-cystine exchanger. Free Radic Biol Med 2015, 86:25-36. 
27. Weng TY, Tsai SA, Su TP: Roles of sigma-1 receptors on mitochondrial functions relevant to neurodegenerative diseases. J Biomed Sci 2017, 24:74.

28. Bernard-Marissal N, Médard JJ, Azzedine H, Chrast R: Dysfunction in endoplasmic reticulummitochondria crosstalk underlies SIGMAR1 loss of function mediated motor neuron degeneration. Brain 2015, 138:875-890.

29. Hedskog L, Pinho CM, Filadi R, Rönnbäck A, Hertwig L, Wiehager B, Larssen P, Gellhaar S, Sandebring $A$, Westerlund $M$, et al: Modulation of the endoplasmic reticulum-mitochondria interface in Alzheimer's disease and related models. Proc Natl Acad Sci U S A 2013, 110:7916-7921.

30. Hu L, Zhang S, Wen H, Liu T, Cai J, Du D, Zhu D, Chen F, Xia C: Melatonin decreases M1 polarization via attenuating mitochondrial oxidative damage depending on UCP2 pathway in prorenin-treated microglia. PloS one 2019, 14:e0212138-e0212138.

31. Lu CW, Lin TY, Wang CC, Wang SJ: $\sigma$-1 Receptor agonist SKF10047 inhibits glutamate release in rat cerebral cortex nerve endings. J Pharmacol Exp Ther 2012, 341:532-542.

32. Zhang S, Hu L, Jiang J, Li H, Wu Q, Ooi K, Wang J, Feng Y, Zhu D, Xia C: HMGB1/RAGE axis mediates stress-induced RVLM neuroinflammation in mice via impairing mitophagy flux in microglia. Journal of Neuroinflammation 2020, 17:15.

33. Ślusarczyk J, Trojan E, Głombik K, Piotrowska A, Budziszewska B, Kubera M, Popiołek-Barczyk K, Lasoń W, Mika J, Basta-Kaim A: Targeting the NLRP3 Inflammasome-Related Pathways via Tianeptine Treatment-Suppressed Microglia Polarization to the M1 Phenotype in LipopolysaccharideStimulated Cultures. International journal of molecular sciences 2018, 19:1965.

34. Paxinos G, Watson CR, Emson PC: AChE-stained horizontal sections of the rat brain in stereotaxic coordinates. J Neurosci Methods 1980, 3:129-149.

35. Grinshpun J, Tveria L, Fleisher-Berkovich S: Differential regulation of prostaglandin synthesis in neonatal rat microglia and astrocytes by somatostatin. European Journal of Pharmacology 2008, 584:312-317.

36. Liu Y, Ma X, Fujioka H, Liu J, Chen S, Zhu X: DJ-1 regulates the integrity and function of ERmitochondria association through interaction with IP3R3-Grp75-VDAC1. Proc Natl Acad Sci U S A 2019, 116:25322-25328.

37. Liu Q, Zhang Y, Liu S, Liu Y, Yang X, Liu G, Shimizu T, Ikenaka K, Fan K, Ma J: Cathepsin C promotes microglia M1 polarization and aggravates neuroinflammation via activation of $\mathrm{Ca}(2+)$-dependent PKC/p38MAPK/NF-KB pathway. J Neuroinflammation 2019, 16:10.

38. Wu J, Prole David L, Shen Y, Lin Z, Gnanasekaran A, Liu Y, Chen L, Zhou H, Chen SRW, Usachev Yuriy $\mathrm{M}$, et al: Red fluorescent genetically encoded $\mathrm{Ca} 2+$ indicators for use in mitochondria and endoplasmic reticulum. Biochemical Journal 2014, 464:13-22.

39. Kanemaru K, Suzuki J, Taiko I, lino M: Red fluorescent CEPIA indicators for visualization of Ca2+ dynamics in mitochondria. Scientific Reports 2020, 10:2835.

40. Jia J, Cheng J, Wang C, Zhen X: Sigma-1 Receptor-Modulated Neuroinflammation in Neurological Diseases. Frontiers in cellular neuroscience 2018, 12:314-314. 
41. Ryskamp DA, Korban S, Zhemkov V, Kraskovskaya N, Bezprozvanny I: Neuronal Sigma-1 Receptors: Signaling Functions and Protective Roles in Neurodegenerative Diseases. Front Neurosci 2019, 13:862.

42. Lannes N, Eppler E, Etemad S, Yotovski P, Filgueira L: Microglia at center stage: a comprehensive review about the versatile and unique residential macrophages of the central nervous system. Oncotarget 2017, 8:114393-114413.

43. Alzayady KJ, Wojcikiewicz RJH: The role of Ca2+ in triggering inositol 1,4,5-trisphosphate receptor ubiquitination. The Biochemical journal 2005, 392:601-606.

44. Bhanumathy CD, Nakao SK, Joseph SK: Mechanism of proteasomal degradation of inositol trisphosphate receptors in CHO-K1 cells. J Biol Chem 2006, 281:3722-3730.

45. Rizzuto R, De Stefani D, Raffaello A, Mammucari C: Mitochondria as sensors and regulators of calcium signalling. Nature Reviews Molecular Cell Biology 2012, 13:566-578.

46. Di Filippo M, Chiasserini D, Tozzi A, Picconi B, Calabresi P: Mitochondria and the Link Between Neuroinflammation and Neurodegeneration. Journal of Alzheimer's Disease 2010, 20:S369-S379.

47. Wilkins HM, Swerdlow RH: Relationships Between Mitochondria and Neuroinflammation: Implications for Alzheimer's Disease. Current topics in medicinal chemistry 2016, 16:849-857.

48. Tondera D, Grandemange S, Jourdain A, Karbowski M, Mattenberger Y, Herzig S, Da Cruz S, Clerc P, Raschke I, Merkwirth C, et al: SLP-2 is required for stress-induced mitochondrial hyperfusion. Embo j 2009, 28:1589-1600.

49. Rossignol R, Gilkerson R, Aggeler R, Yamagata K, Remington SJ, Capaldi RA: Energy Substrate Modulates Mitochondrial Structure and Oxidative Capacity in Cancer Cells. Cancer Research 2004, 64:985-993.

50. Park MK, Ashby MC, Erdemli G, Petersen OH, Tepikin AV: Perinuclear, perigranular and subplasmalemmal mitochondria have distinct functions in the regulation of cellular calcium transport. Embo j 2001, 20:1863-1874.

51. Youle RJ, Karbowski M: Mitochondrial fission in apoptosis. Nat Rev Mol Cell Biol 2005, 6:657-663.

52. Rambold AS, Pearce EL: Mitochondrial Dynamics at the Interface of Immune Cell Metabolism and Function. Trends in Immunology 2018, 39:6-18.

53. Zhou R, Yazdi AS, Menu P, Tschopp J: A role for mitochondria in NLRP3 inflammasome activation. Nature 2011, 469:221-225.

54. Swanson KV, Deng M, Ting JPY: The NLRP3 inflammasome: molecular activation and regulation to therapeutics. Nature Reviews Immunology 2019, 19:477-489.

55. McCormack JG, Halestrap AP, Denton RM: Role of calcium ions in regulation of mammalian intramitochondrial metabolism. Physiol Rev 1990, 70:391-425.

56. Hansford RG: Physiological role of mitochondrial Ca2+ transport. Journal of Bioenergetics and Biomembranes 1994, 26:495-508. 
57. Youle RJ, van der Bliek AM: Mitochondrial fission, fusion, and stress. Science (New York, NY) 2012, 337:1062-1065.

58. Silva Ramos E, Larsson NG, Mourier A: Bioenergetic roles of mitochondrial fusion. Biochim Biophys Acta 2016, 1857:1277-1283.

59. Pagliuso A, Cossart $P$, Stavru $F$ : The ever-growing complexity of the mitochondrial fission machinery. Cellular and molecular life sciences : CMLS 2018, 75:355-374.

60. Gomes LC, Di Benedetto G, Scorrano L: During autophagy mitochondria elongate, are spared from degradation and sustain cell viability. Nat Cell Biol 2011, 13:589-598.

61. Wu J, Li X, Zhu G, Zhang Y, He M, Zhang J: The role of Resveratrol-induced mitophagy/autophagy in peritoneal mesothelial cells inflammatory injury via NLRP3 inflammasome activation triggered by mitochondrial ROS. Exp Cell Res 2016, 341:42-53.

62. Nakahira K, Haspel JA, Rathinam VA, Lee SJ, Dolinay T, Lam HC, Englert JA, Rabinovitch M, Cernadas $\mathrm{M}, \mathrm{Kim} \mathrm{HP}$, et al: Autophagy proteins regulate innate immune responses by inhibiting the release of mitochondrial DNA mediated by the NALP3 inflammasome. Nat Immunol 2011, 12:222-230.

63. Shimada K, Crother TR, Karlin J, Dagvadorj J, Chiba N, Chen S, Ramanujan VK, Wolf AJ, Vergnes L, Ojcius DM, et al: Oxidized mitochondrial DNA activates the NLRP3 inflammasome during apoptosis. Immunity 2012, 36:401-414.

64. Krishnan SM, Ling YH, Huuskes BM, Ferens DM, Saini N, Chan CT, Diep H, Kett MM, Samuel CS, Kemp-Harper BK, et al: Pharmacological inhibition of the NLRP3 inflammasome reduces blood pressure, renal damage, and dysfunction in salt-sensitive hypertension. Cardiovascular Research 2018, 115:776-787.

65. Liu Q, Zhang D, Hu D, Zhou X, Zhou Y: The role of mitochondria in NLRP3 inflammasome activation. Mol Immunol 2018, 103:115-124.

66. Sata Y, Head GA, Denton K, May CN, Schlaich MP: Role of the Sympathetic Nervous System and Its Modulation in Renal Hypertension. Frontiers in Medicine 2018, 5.

67. Mahfoud F, Lüscher TF, Andersson B, Baumgartner I, Cifkova R, DiMario C, Doevendans P, Fagard R, Fajadet J, Komajda M, et al: Expert consensus document from the European Society of Cardiology on catheter-based renal denervationt. European Heart Journal 2013, 34:2149-2157.

\section{Figures}



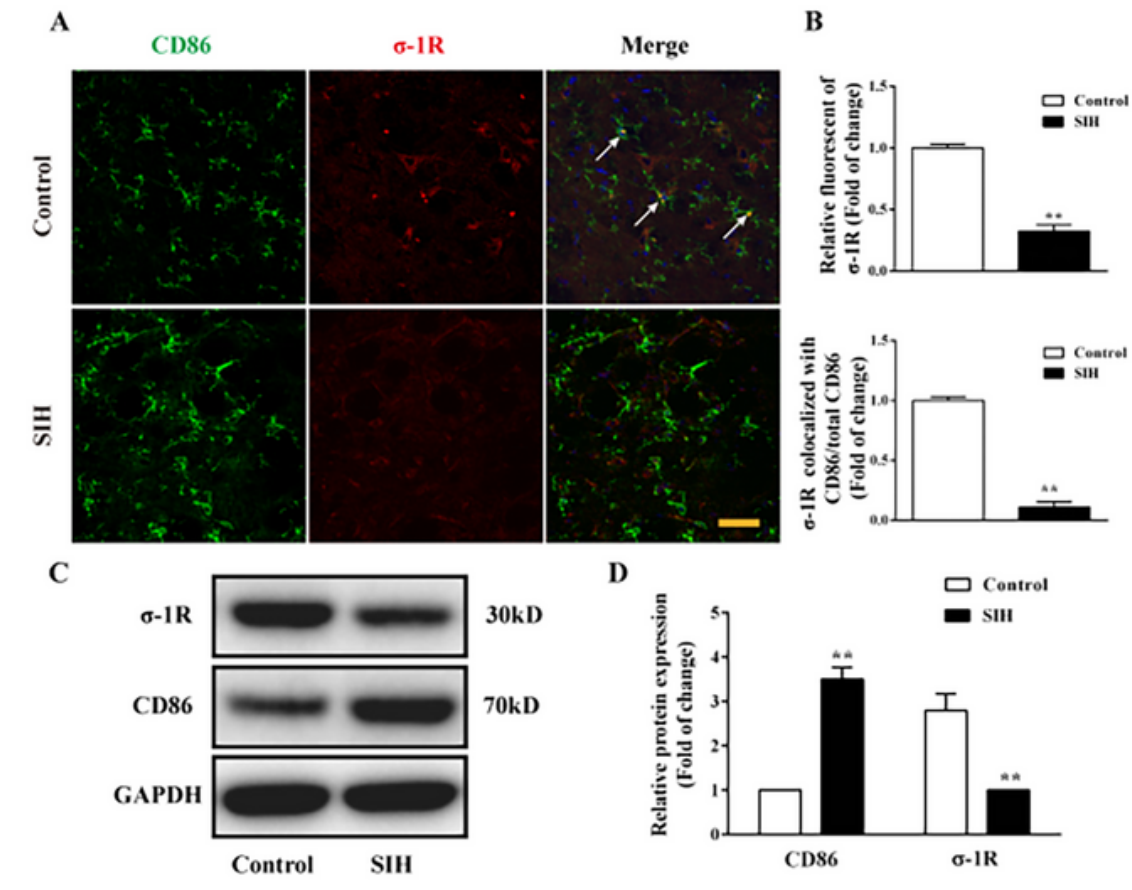

E
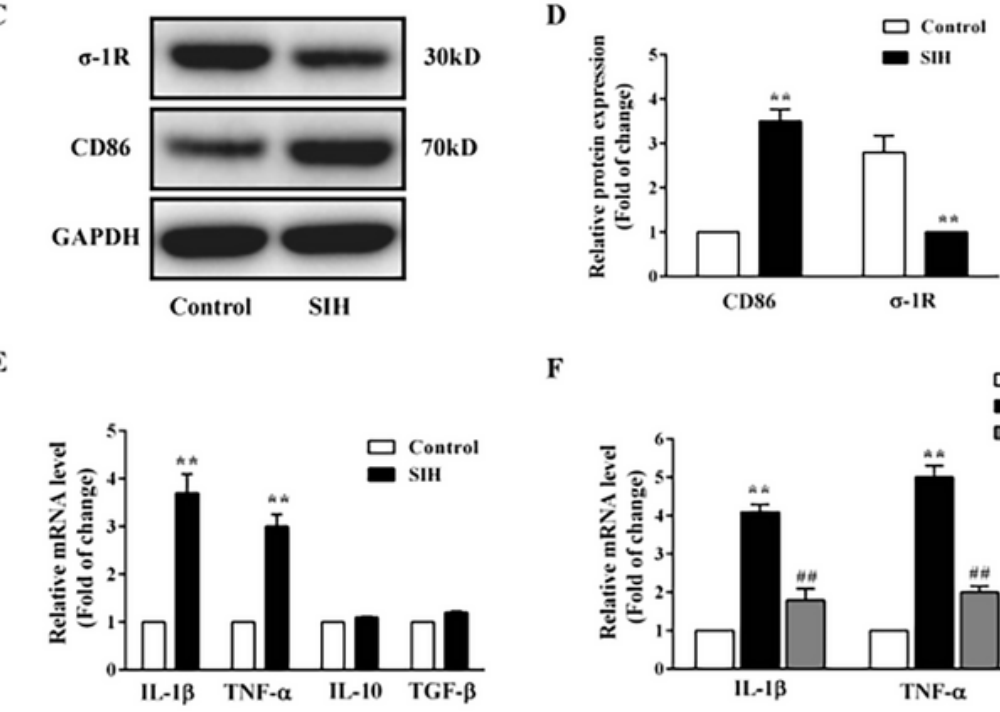

F

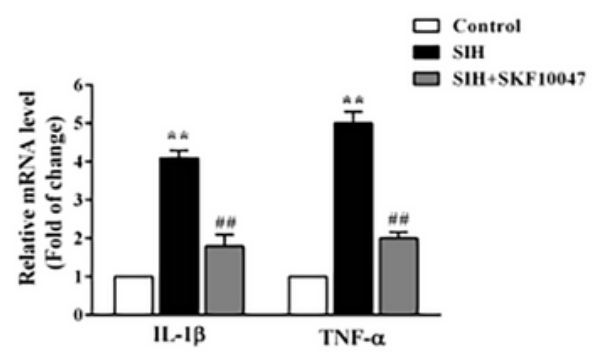

G

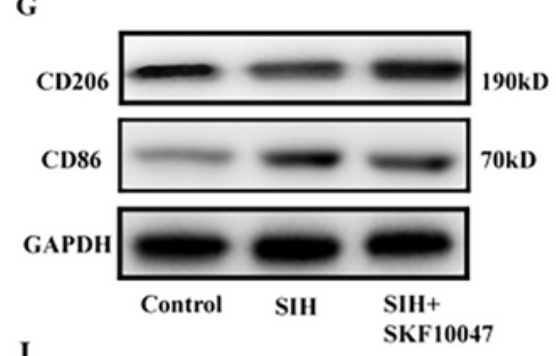

H
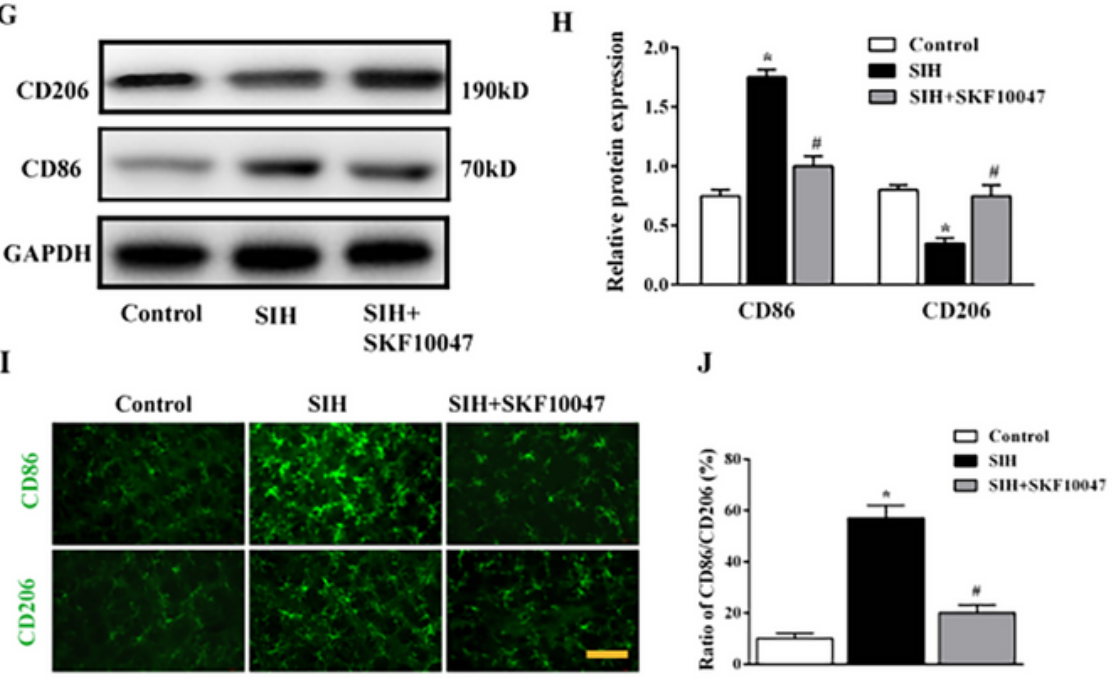

\section{Figure 1}

Stress reduced $\sigma-1 \mathrm{R}$ expression and enhanced neuroinflammation in the RVLM of rats. A Double immunofluorescence showed colocalization of CD86 and $\sigma-1 \mathrm{R}$ in the RVLM of control and SIH groups. Scale bar $=20 \mu \mathrm{m}$. B Quantitative analysis of fluorescence intensity of $\sigma-1 \mathrm{R} / \mathrm{CD} 86$ in the RVLM of rats. CD Representative immunoblot bands and quantitative analysis shows the expression of $\sigma-1 R$ and CD86. E-F qRT-PCR of RVLM showed the expression of TNF-a and IL-1 $\beta$ in different group rats. G-H 
Representative immunoblot bands and respective analysis of CD86 (M1) and CD206 (M2) in the RVLM. I Immunofluorescent detection of M1 and/ or M2 phenotype microglia in the RVLM. Scale bar $=20 \mu \mathrm{m} . \mathrm{J}$ Ratio of CD86 to CD206 expressed in percentage. Data are presented as mean \pm SEM. $n=6, \star \star P<0.01$ vs. control, \#\#P $<0.01$ vs. SIH, *P $<0.05$ vs. control, \#P $<0.05$ vs. SIH.

A

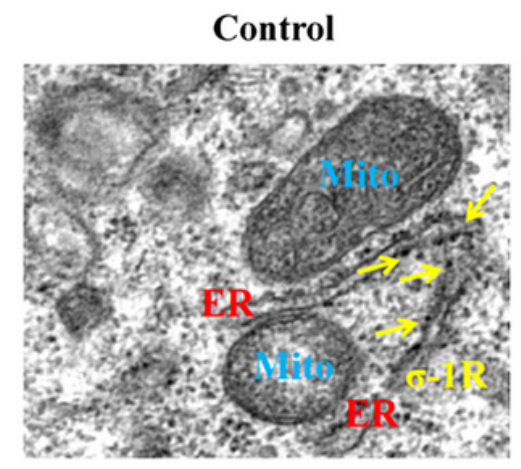

SIH

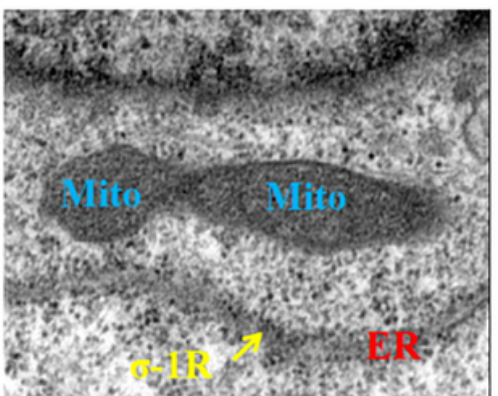

SIH+SKF10047

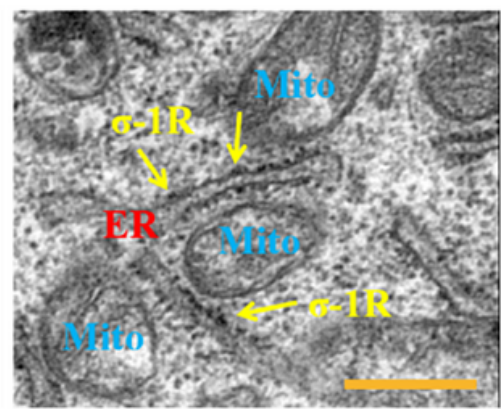

B

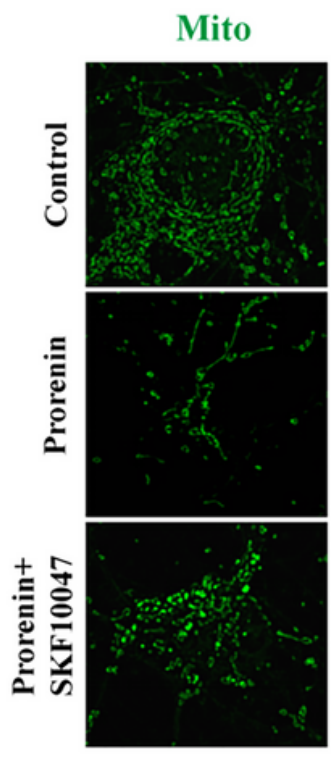

ER
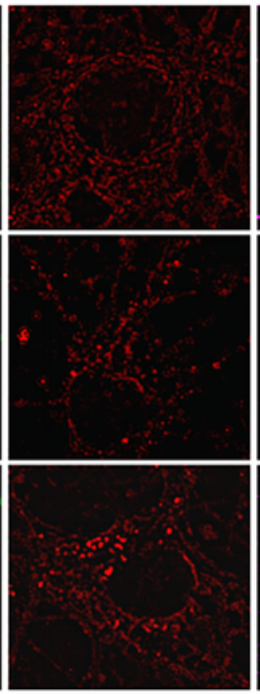

$\sigma 1-\mathrm{R}$

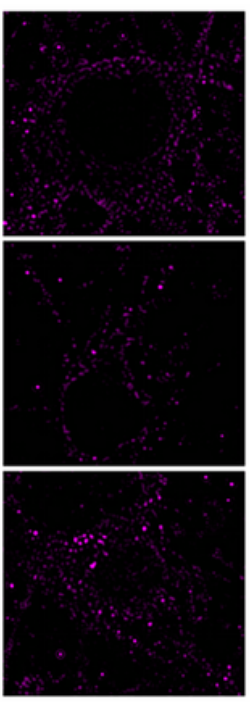

Merge
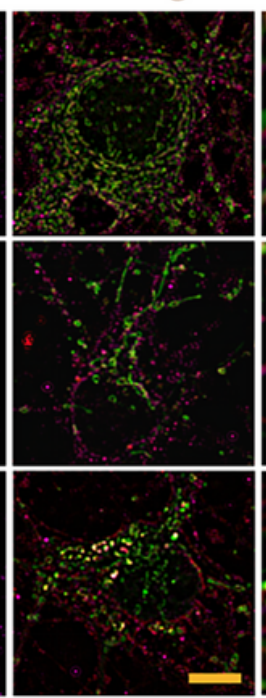

C

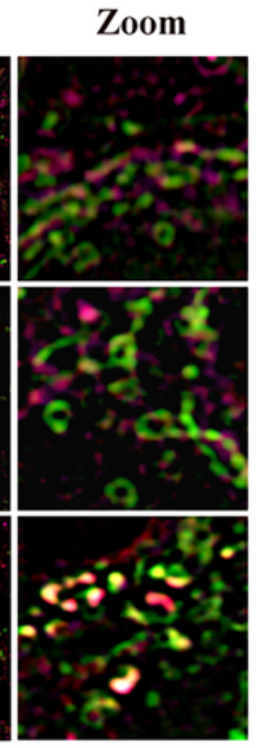

D
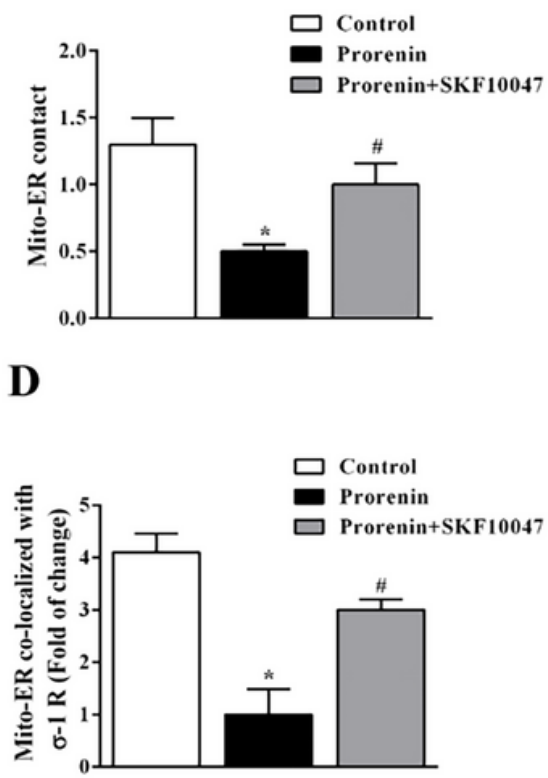

\section{Figure 2}

$\sigma-1 \mathrm{R}$ was predominantly expressed at the mitochondrial-associated endoplasmic reticulum membrane (MAMs). A Transmission-electron microscopy indicated $\sigma-1 \mathrm{R}$ was predominantly expressed and distributed at the MAMs (yellow arrows). Scale bar $=600 \mathrm{~nm}$. B Trichome immunofluorescence staining was used to label mmitochondria (green), ER (red) and $\sigma-1 R$ (purple), merged immunofluorescence is used to visualize MAMs/ $\sigma-1 R$ colocalization. Scale bar $=2 \mu \mathrm{m}$. C-D Quantitative analysis of Mito-ER contact (MAMs) based on fluorescence intensity. Data are presented as mean \pm SEM. $n=6, * P<0.05$ vs. control, \#P< 0.05 vs. prorenin. 
A

Control
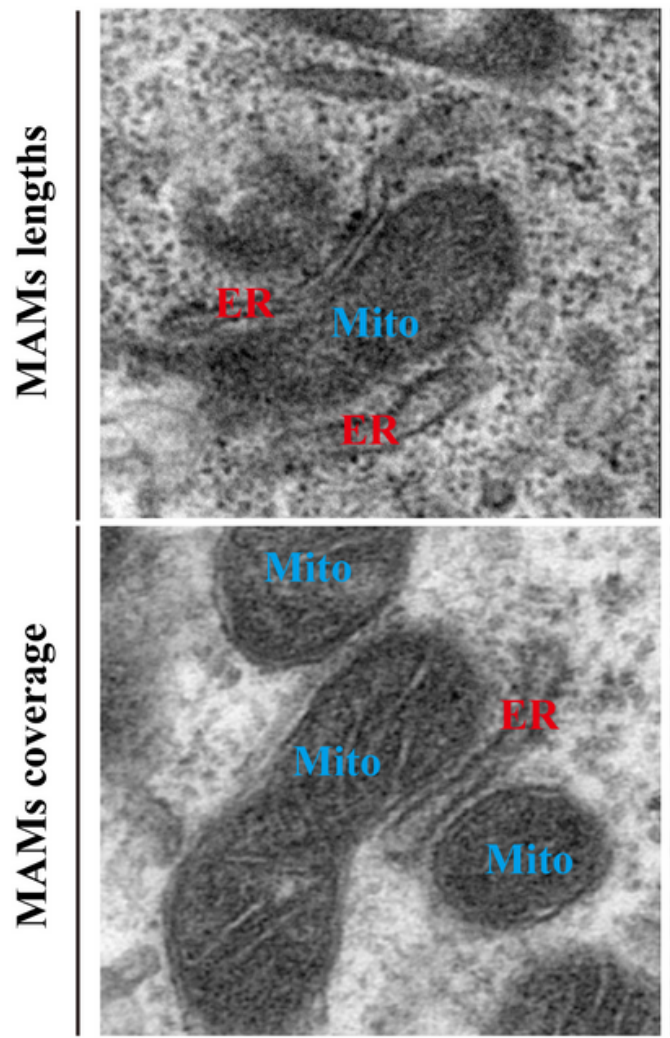

Prorenin
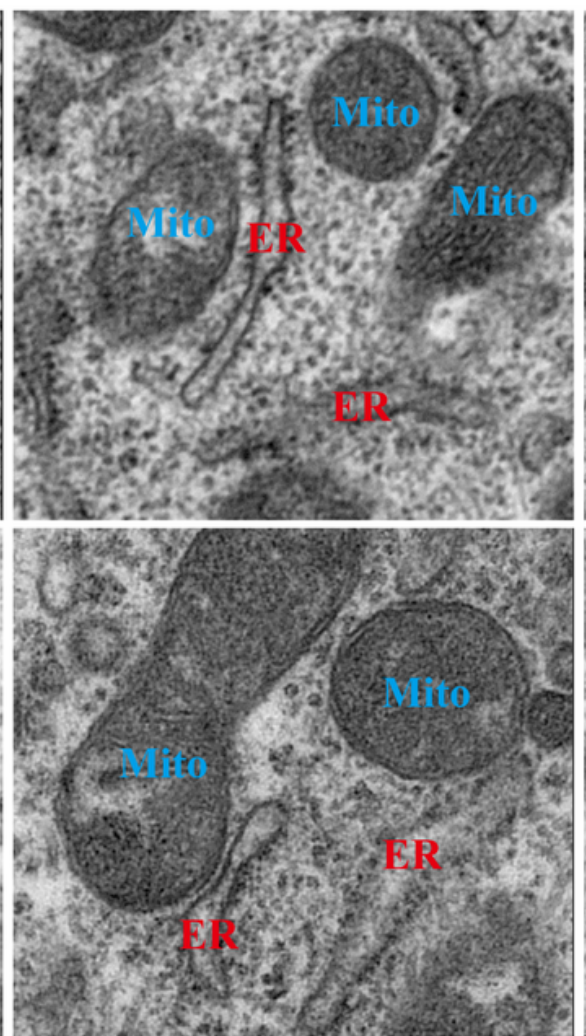

Prorenin+SKF10047

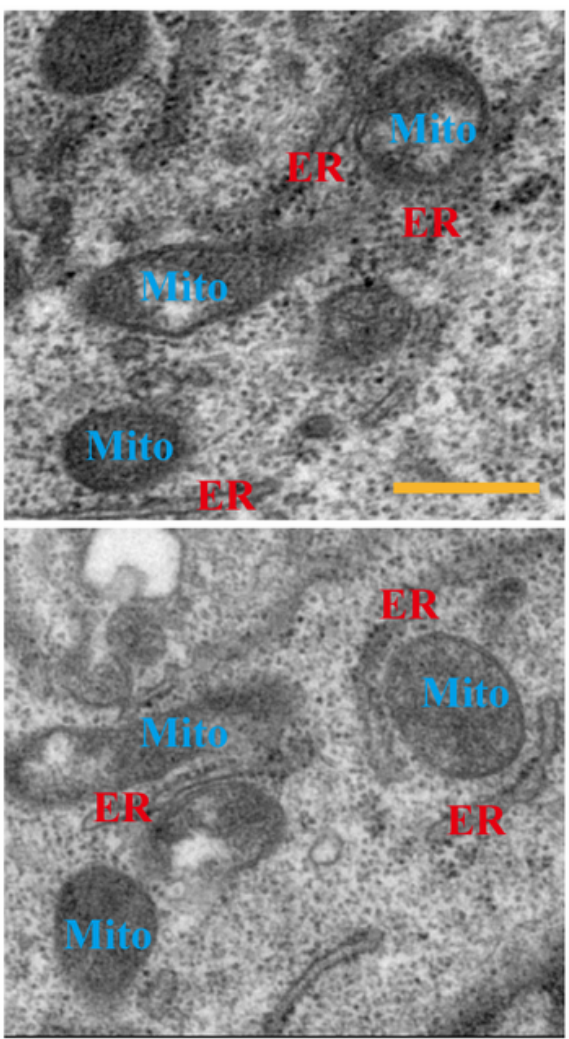

B

C

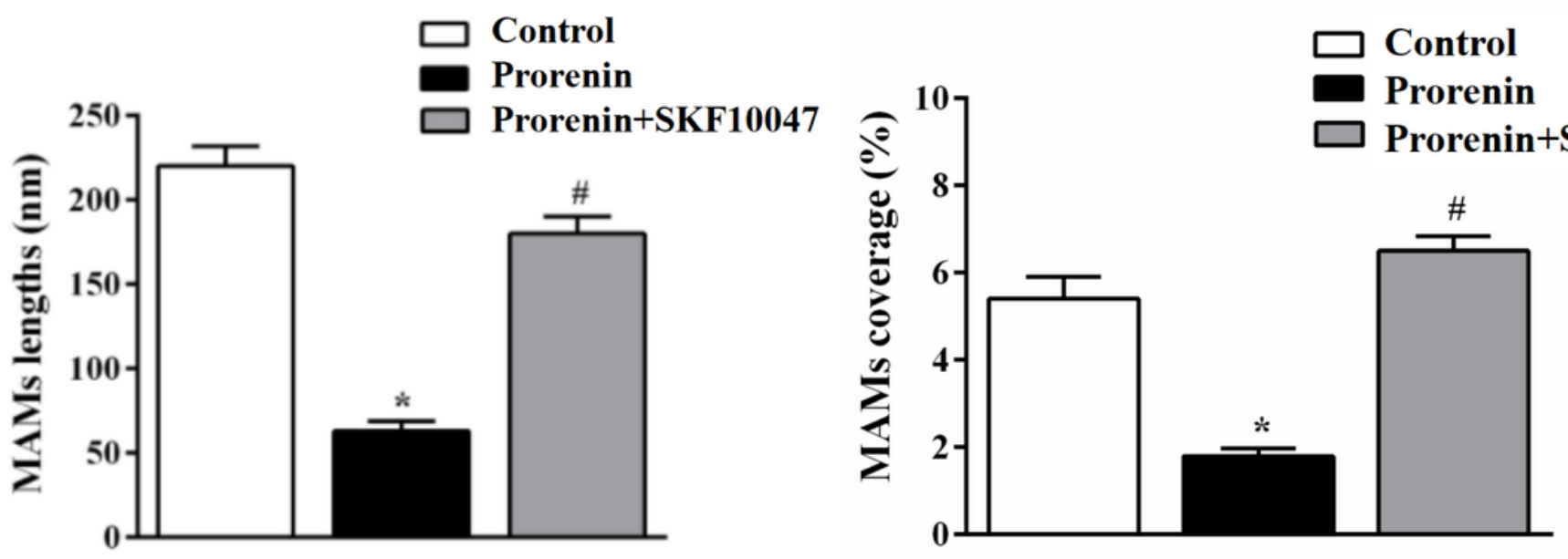

Figure 3

Immune transmission electron microscopy showed that activation of $\sigma-1 \mathrm{R}$ promotes formation and association of MAMs. A Immune-electron microscopy showing MAMs coverage and length in control, rorenin, and prorenin+SKF10047 group. Scale bar $=500 \mathrm{~nm}$. B MAMs length were measured. C MAMs coverage was calculated and presented in percentage. Data are presented as mean $\pm S E M . n=6, * P$ $<0.05$ vs. control, \#P $<0.05$ vs. prorenin. 
A

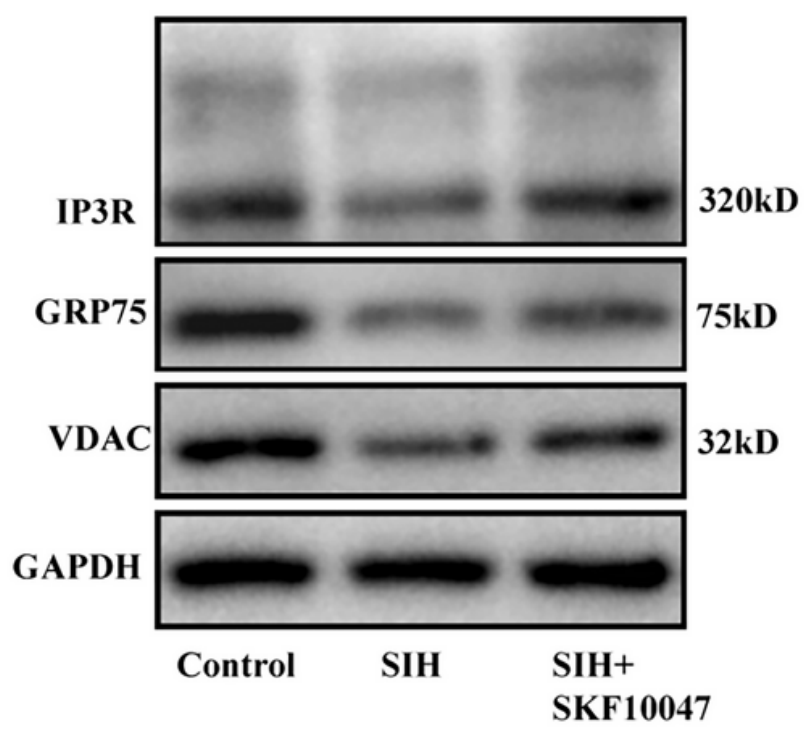

C

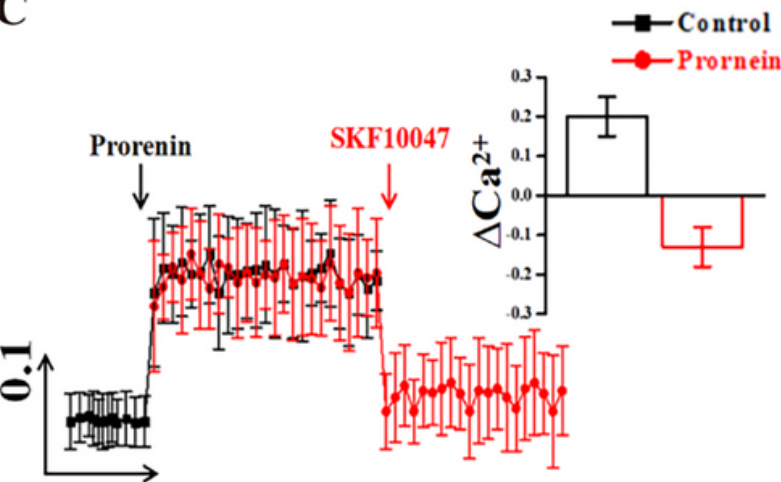

50s ER-LAR-GECO1.2

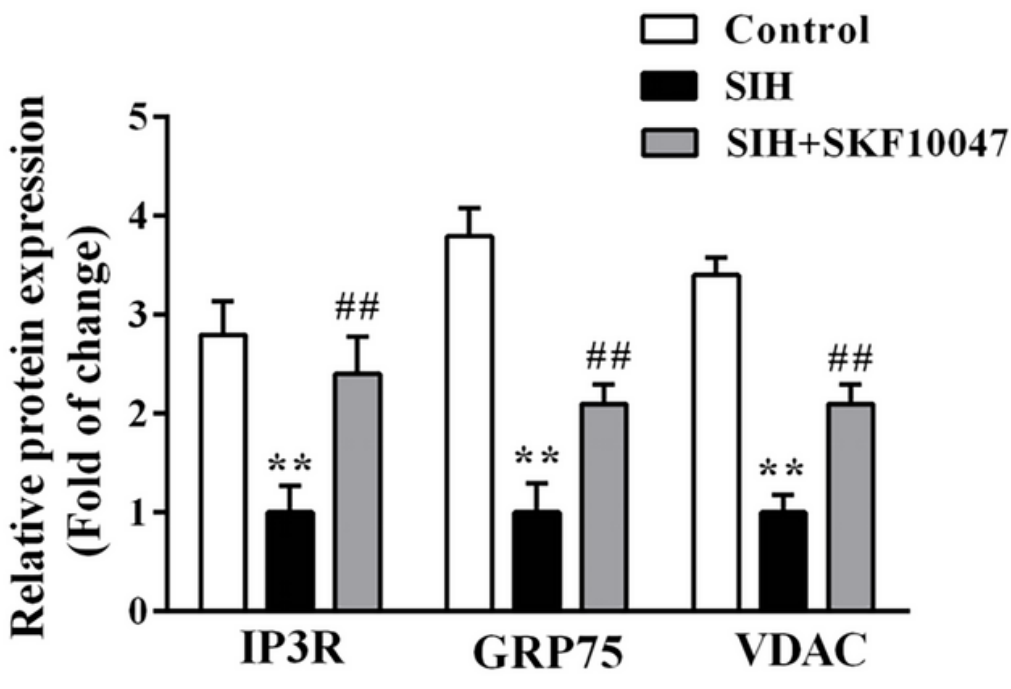

\section{Figure 4}

Effects of $\sigma-1 R$ activation on the components' expression of IP3R-GRP75-VDAC transport complex, rescues the MAMs-dependent $\mathrm{Ca} 2+$ signalling between the two organelles. A-B Representative immunoblot and quantitative analysis showing that IP3R, GRP75 and VDAC expressions. C GECO analysis indicates changes in intra-ER Ca2+ concentration before and after SKF10047 treatment. D CEPIA4 analysis indicates changes in intra-mitochondrial Ca2+ concentration before and after SKF10047 treatment. Data are presented as mean \pm SEM. $n=6,{ }^{\star} \mathrm{P}<0.01$ vs. control, \#\#P<0.01 vs. SIH. 
A

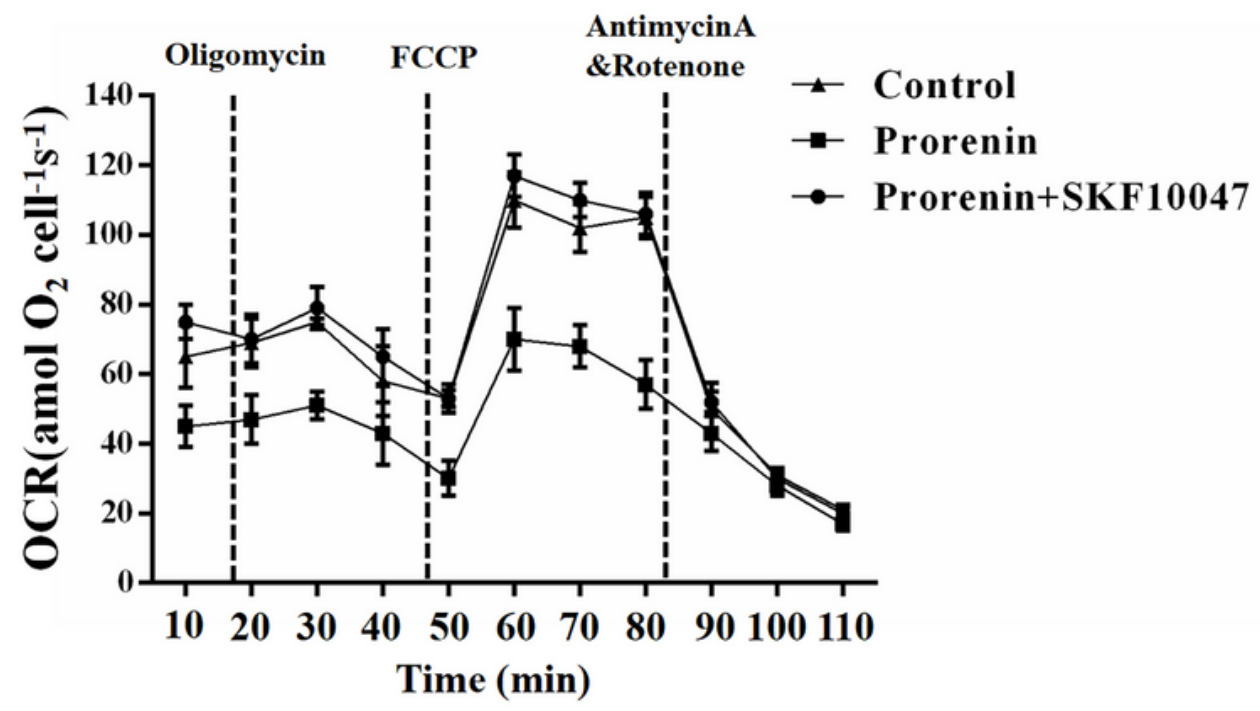

$\mathbf{B}$
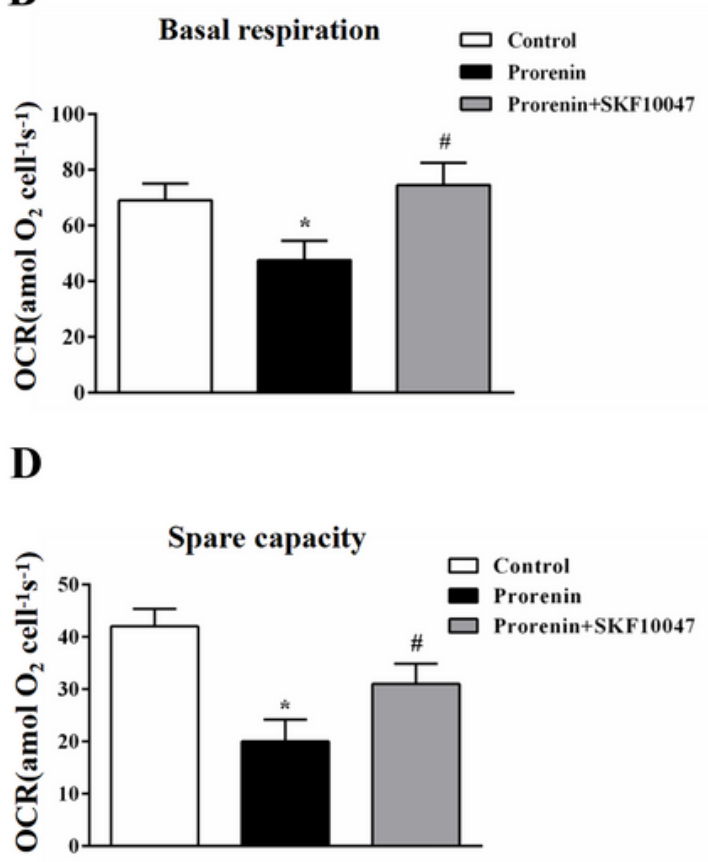

$\mathbf{F}$

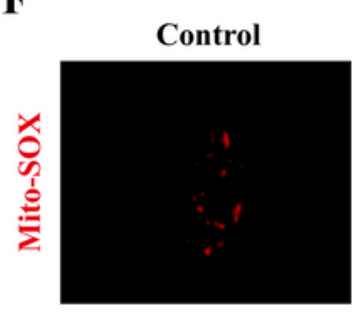

C

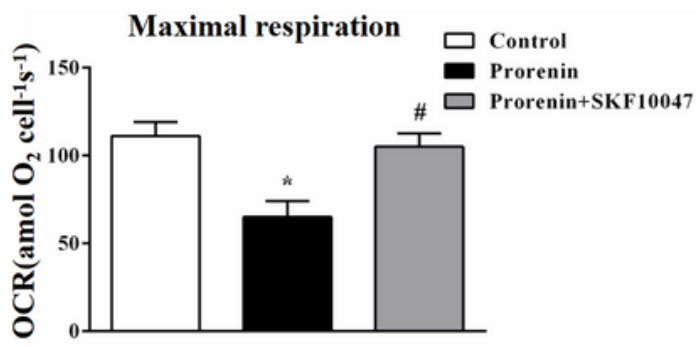

$\mathbf{E}$

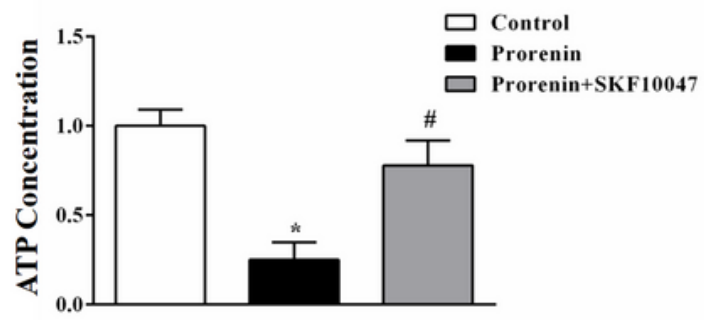

$20 \mu \mathrm{m}$

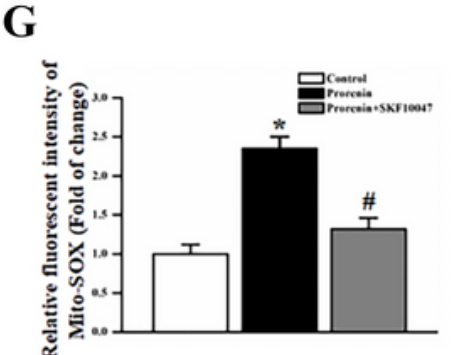

Figure 5

Mitochondrial respiratory function measurement by Seahorse cell metabolometer and mitoROS measurement. A Metabolic parameters of BV2 cells cultured in vitro with or without $100 \mu \mathrm{M} / \mathrm{L}$ SKF10047 after $24 \mathrm{~h}$ prorenin treated. B The oxygen consumption rate (OCR) of BV-2 cells in basal respiration. $\mathrm{C}$ OCR in maximal respiration. D energy potential. E ATP concentration. F Representative image of mitochondrial ROS production in the control, prorenin, and prorenin+SKF10047 group is quantified using 
Mito-SOX kits. G MitoROS quantification as indicated by the fluorescent intensity in the different groups. Data are presented as mean \pm SEM. $n=6,{ }^{*}<<0.05$ vs. control, $\# \mathrm{P}<0.05$ vs. prorenin.

A
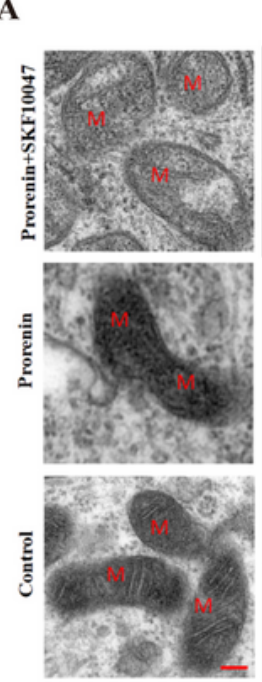

D

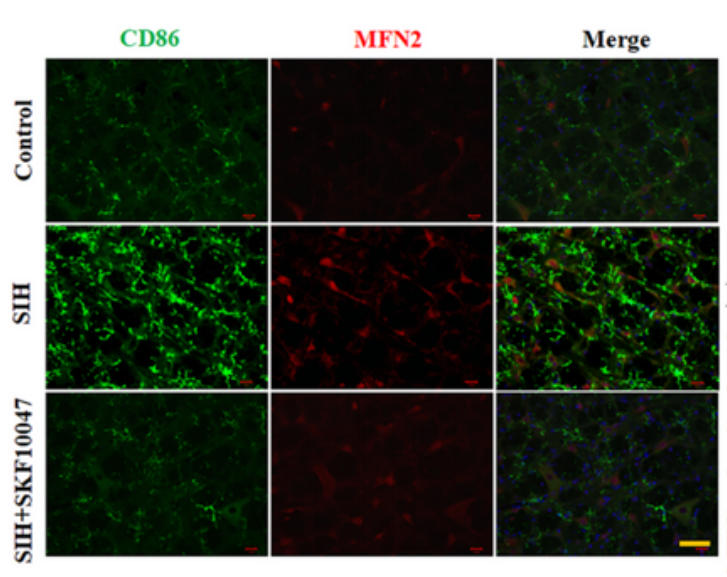

G

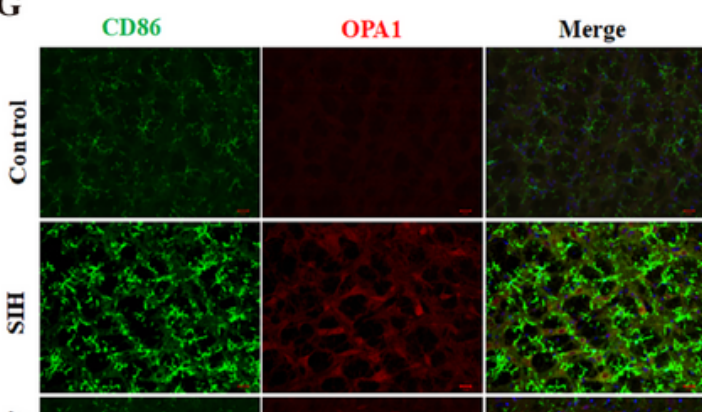

옹

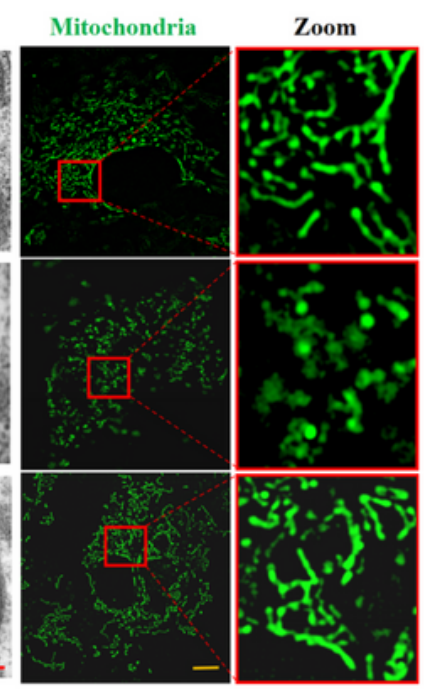

B

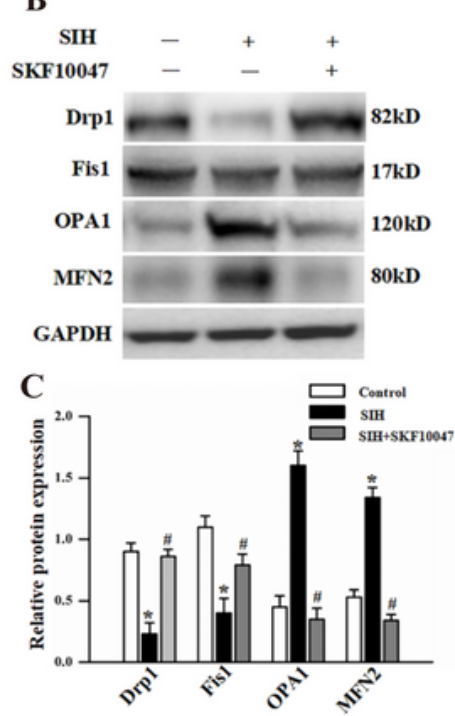

E

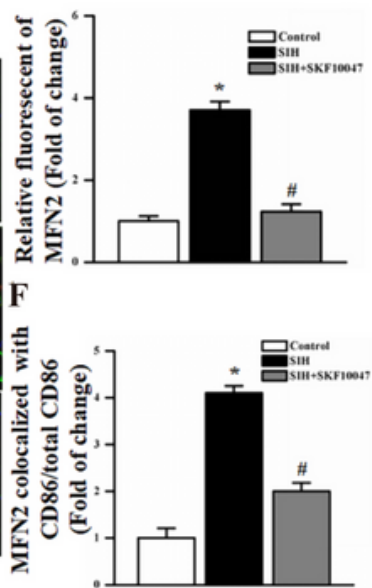

$\mathrm{H}$
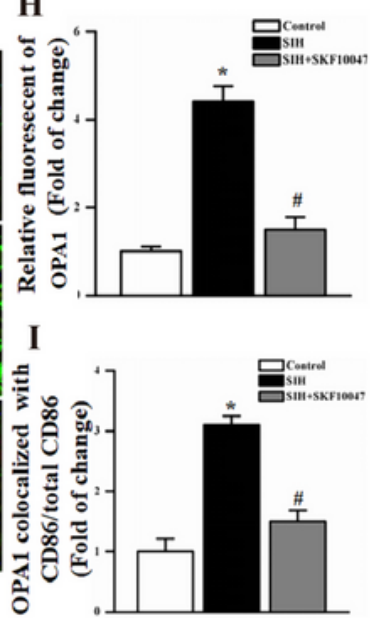

Figure 6

$\sigma-1 R$ reduces mitochondrial hyperfusion and microglial $M 1$ polarization in stressed microglia. A TEM images showed that prorenin stimulated mitochondrial hyperfusion as indicated by fused, elongated and enlarged network (left); super-resolution microscopy showed that SKF10047 treatment prevented 
mitochondria from hyperfusion as shown relatively normal size and shape mitochondria (middle and right). B,C Representative immunoblots and quantitative analysis showing that expressions of fusion proteins (MFN2, OPA1) and fission proteins (Drp1, Fis 1) in different groups. D-F Representative images and quantitative analysis showing colocalization of CD86 (green) and MFN2 (red) in the RVLM. G-I Representative images and quantitative analysis showing colocalization of CD86 (green) and OPA1 (red) in the RVLM. Data represent mean \pm SEM. $n=6,{ }^{*} P<0.05$ vs. control, $\# P<0.05$ vs. SIH. Scale bar $=20$ $\mu \mathrm{m}$ in (D) and (G).

A

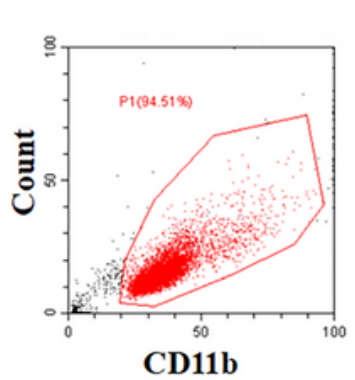

CD11b
B

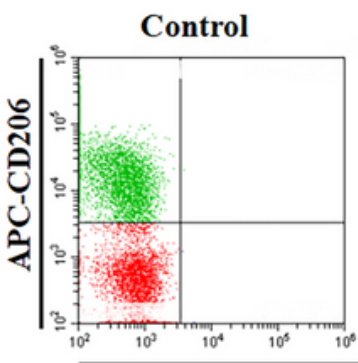

Prorenin+SKF10047

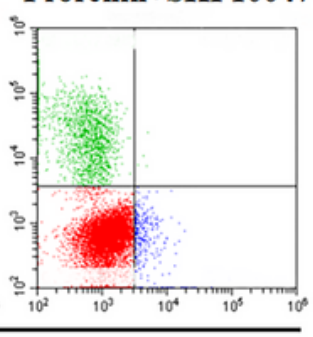

PE-CD86
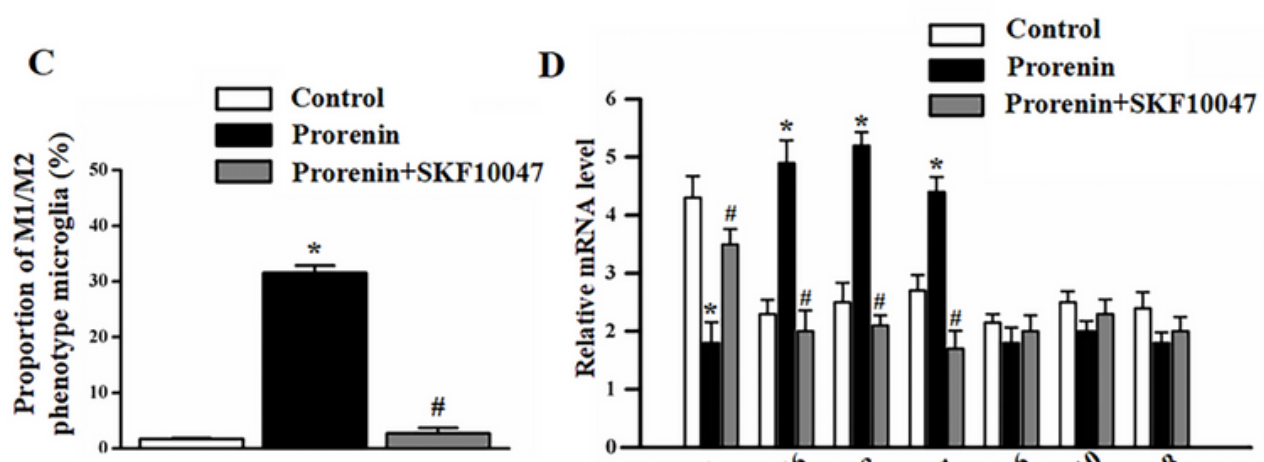

E

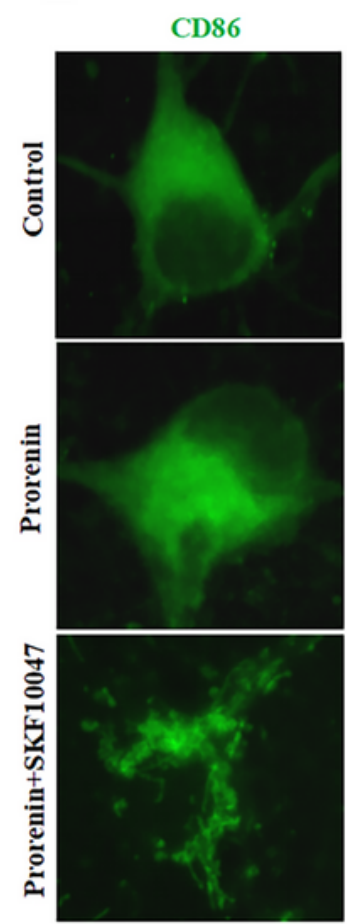

NLRP3

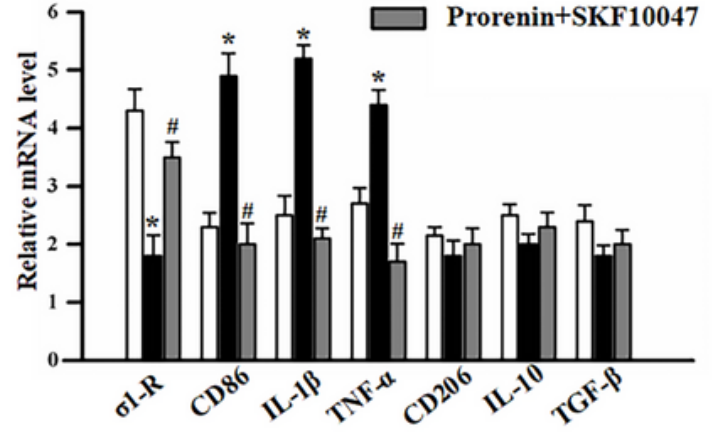

F

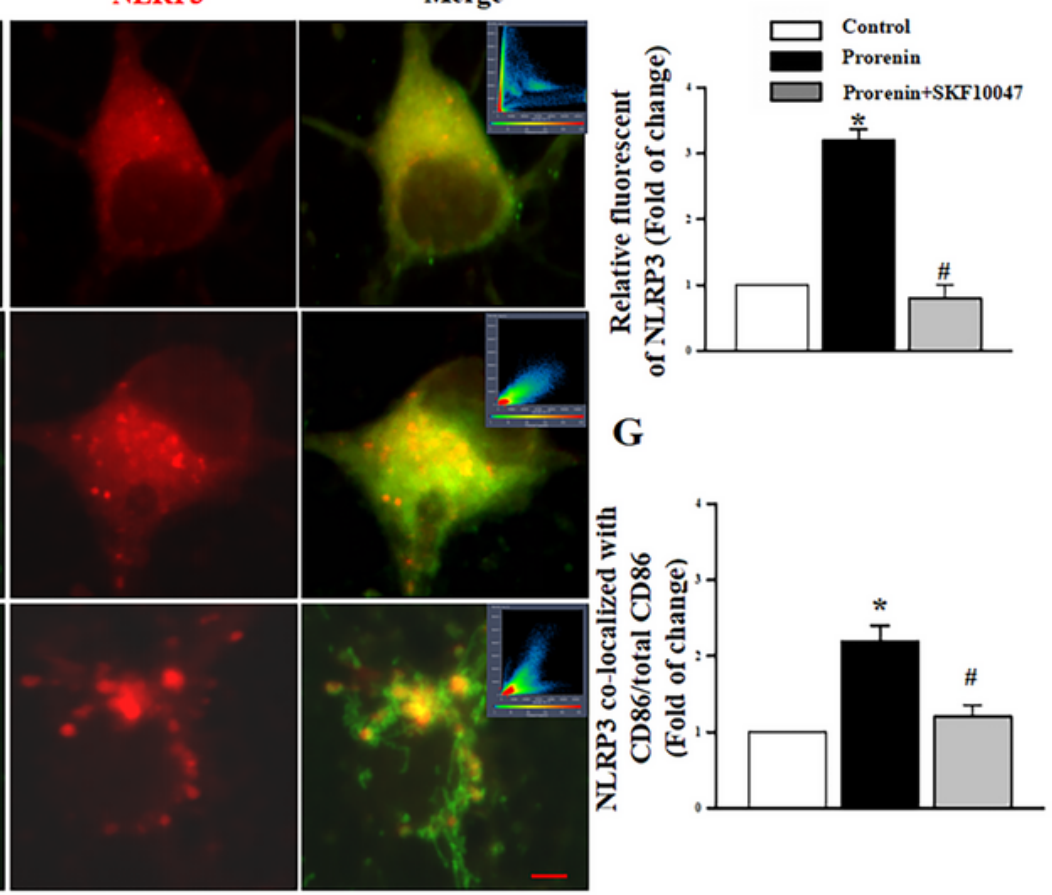


Figure 7

SKF10047 treatment decreased the ratio of M1/M2 polarization and NLRP3 inflammasomes in prorenintreated primary microglia. A Flow cytometry analysis showed that the purity of primary microglia was greater than $90 \%$. B,C FACS analysis indicated the ratio of immunophenotypic transformation of M1 to M2 microglia. D qRT-PCR analysis indicating mRNA levels of $\sigma-1 R, C D 86, C D 206$, pro-inflammatory cytokines (TNF- $\alpha$, IL- $\beta$ ) and anti-inflammatory cytokines (IL-10, TGF- $\beta$ ). E-G Representative images and quantitative analysis showing colocalization of CD86(green) and NLRP3(red) in the RVLM. NLRP3 immunofluorescence and NLRP3/CD86 colocalization. Data represent mean \pm SEM. $n=5, * P<0.05$ vs. control, \#P $<0.05$ vs. prorenin.

A

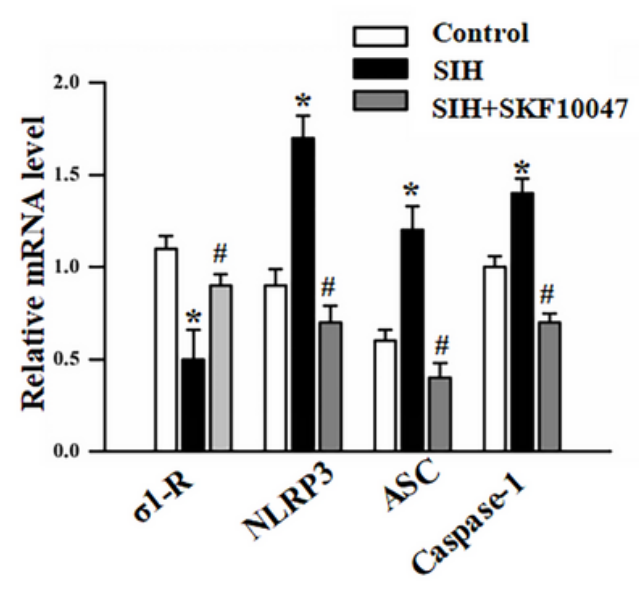

B

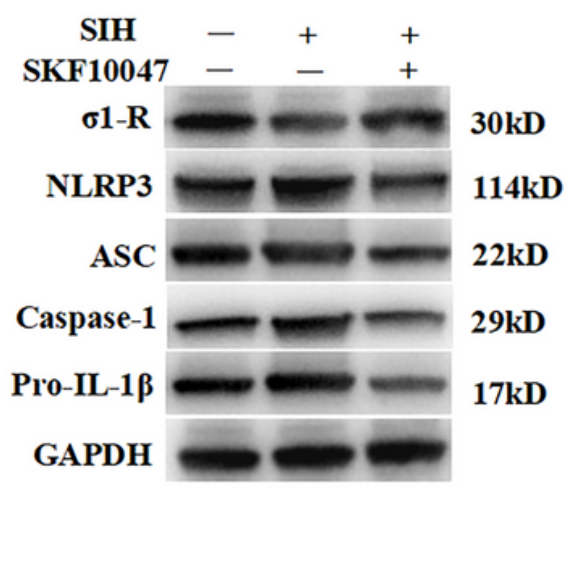

C

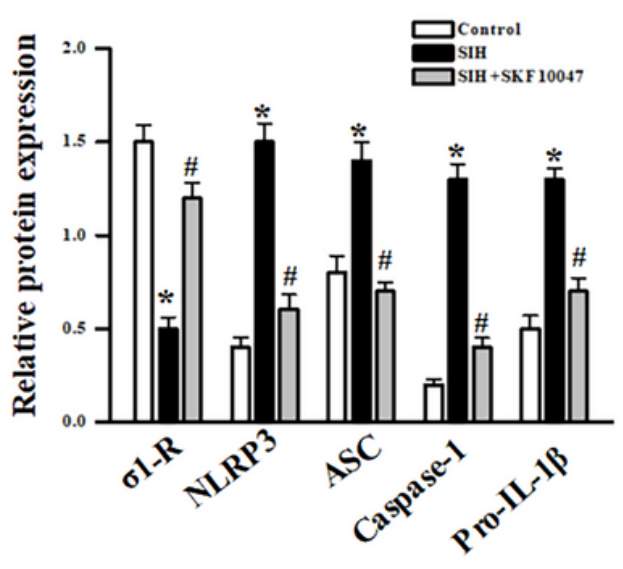

E

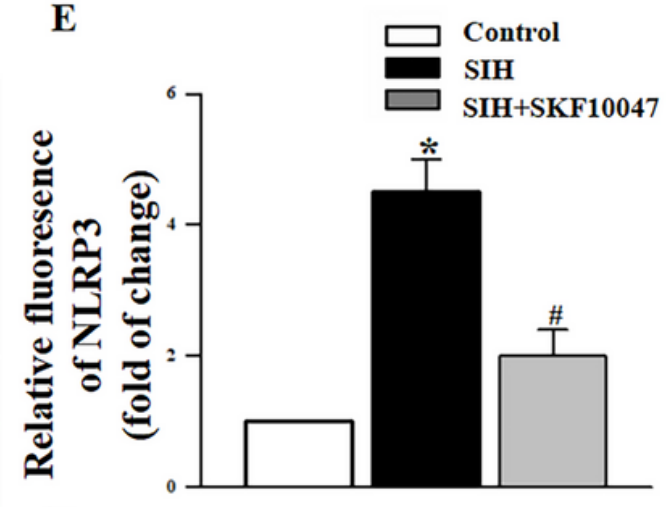

$F$

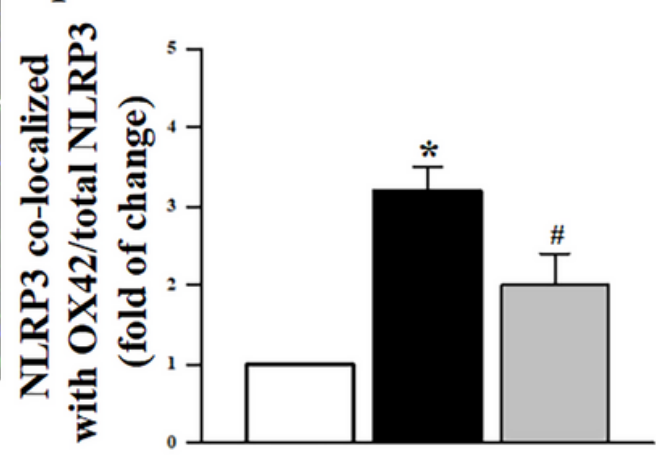

Figure 8 
In-vivo $\triangle S K F 10047$ treatment preventing downstream NLRP3 activation and M1 microglia polarization. A$C$ Relative mRNA level, representative western blot, and quantitative analysis revealing the expressions of $\sigma-1 \mathrm{R}, \mathrm{NLRP} 3$, ASC, Caspase- 1 and Pro-IL-1 $\beta$ in SIH and SIH+SKF10047 group. D Representative images showing that NLRP3 (red) colocalized with the M1 microglia marker (CD86+, green). Scale bar $=20 \mu \mathrm{m}$. E, F Relative fluorescence intensity calculation of NLRP3-immunoreactivity with or without OX42immunoreactivity in control, SIH and SIH+SKF10047 group. Data are presented as mean \pm SEM. $n=6,{ }^{*} P$ $<0.05$ vs. control, \#P $<0.05$ vs SIH.

A

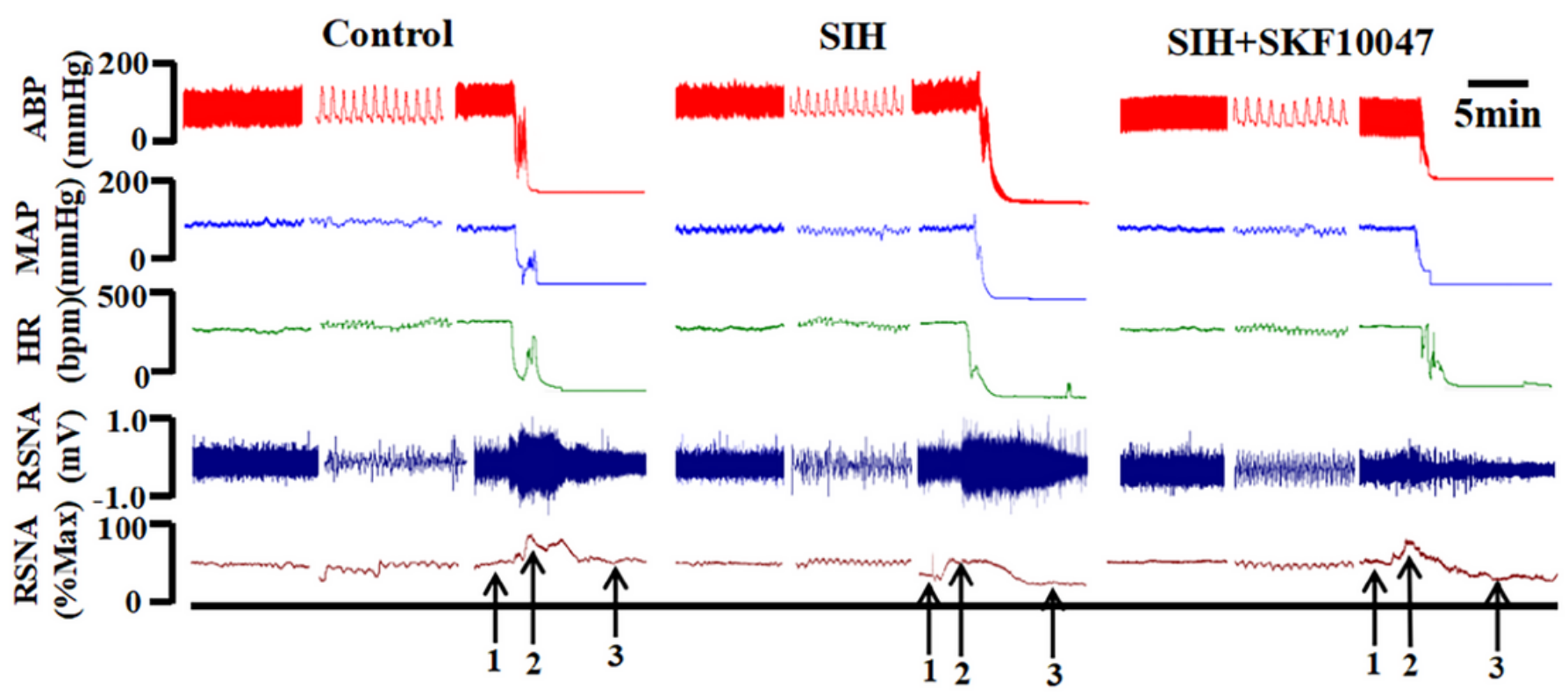

B

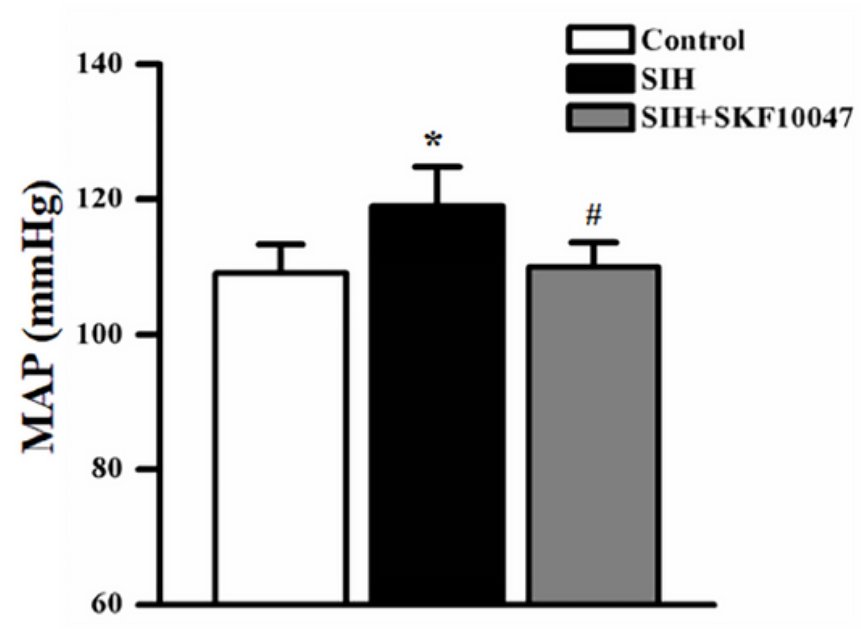

C

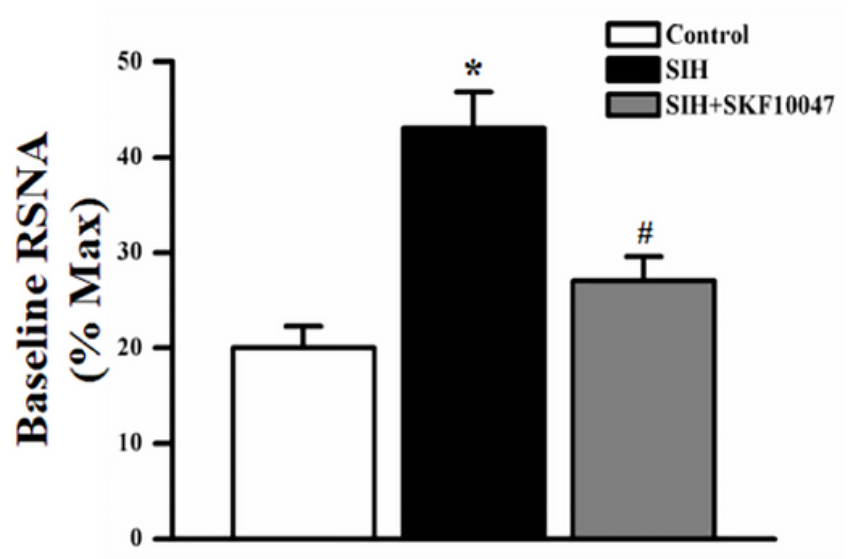

\section{Figure 9}

$\sigma-1 \mathrm{R}$ activation ameliorates sympathetic hyperactivity and stress-induced hypertension in rats. A Representative hemodynamic trace demonstrating the effect of stress and SKF10047 treatment on ABP, MAP, HR, RSNA and \% MAX RSNA (1, basal RSNA; 2, maximum RSNA; 3, noise level) B Statistical data for MAP in different groups. Stress significantly elevated MAP, the MAP increment is attenuated by 
SKF10047. C Statistical data for Baseline RSNA in different groups. Data are presented as mean \pm SEM. ${ }^{*} \mathrm{P}<0.05$ vs. control, \#P $<0.05$ vs $\mathrm{SIH}, \mathrm{n}=6$ /group. ABP, arterial blood pressure; MAP, mean arterial pressure; HR, heart rate; RSNA, renal sympathetic nerve activity.

\section{Supplementary Files}

This is a list of supplementary files associated with this preprint. Click to download.

- graphicalabstract.png 